See discussions, stats, and author profiles for this publication at: https://www.researchgate.net/publication/340418024

\title{
Canard-induced complex oscillations in an excitatory network
}

Article in Journal of Mathematical Biology · April 2020

DOI: $10.1007 / 500285-020-01490-1$

CITATION

1

5 authors, including:

Toni Guillamon Grabolosa

Universitat Politècnica de Catalunya

61 PUBLICATIONS 647 CITATIONS

SEE PROFILE

Some of the authors of this publication are also working on these related projects:

Estimation of Synaptic Conductance in the Spiking Regime View project

Circadian control of reproductive hormons in female rat View project
Florida State University

60 PUBLICATIONS 1,237 CITATIONS

SEE PROFILE 


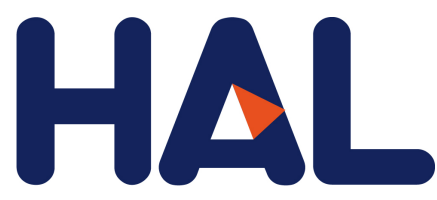

archives-ouvertes

\section{Canard-induced complex oscillations in an excitatory network}

Elif Köksal Ersöz, Mathieu Desroches, Antoni Guillamon, John Rinzel, Joel

Tabak

\section{- To cite this version:}

Elif Köksal Ersöz, Mathieu Desroches, Antoni Guillamon, John Rinzel, Joel Tabak. Canard-induced complex oscillations in an excitatory network. Journal of Mathematical Biology, Springer Verlag (Germany), 2020, 10.1007/s00285-020-01490-1 . hal-01939157v2

\section{HAL Id: hal-01939157 \\ https://hal.inria.fr/hal-01939157v2}

Submitted on 31 Mar 2020

HAL is a multi-disciplinary open access archive for the deposit and dissemination of scientific research documents, whether they are published or not. The documents may come from teaching and research institutions in France or abroad, or from public or private research centers.
L'archive ouverte pluridisciplinaire HAL, est destinée au dépôt et à la diffusion de documents scientifiques de niveau recherche, publiés ou non, émanant des établissements d'enseignement et de recherche français ou étrangers, des laboratoires publics ou privés. 


\title{
Canard-induced complex oscillations in an excitatory network
}

\author{
Elif Köksal Ersöz • Mathieu Desroches • \\ Antoni Guillamon • John Rinzel . \\ Joël Tabak
}

Received: date / Accepted: date

\begin{abstract}
In Neuroscience, mathematical modelling involving multiple spatial and temporal scales can unveil complex oscillatory activity such as excitability, subthreshold oscillations, spiking or bursting. While the number of slow and fast variables and the geometry of the system determine the type of the complex oscillations, canard structures define boundaries between them. In this study, we use geometric singular perturbation theory to identify and characterise boundaries between different dynamical regimes of multiple-timescale firing rate models of the developing spinal cord. These rate models are either three or four dimensional with state variables chosen within an overall group of two slow and two fast variables. The fast subsystem corresponds to a recurrent excitatory network with fast activity-dependent synaptic depression, and the slow variables represent the cell firing threshold and slow activity-dependent synaptic depression, respectively. We start by studying canard-induced bursting and mixed-mode oscillations in two different three-dimensional rate models. Then, in the full four-dimensional model we show that a canard-mediated slow passage creates dynamics that combine these complex oscillations and
\end{abstract}

\footnotetext{
E. Köksal Ersöz
}

MathNeuro Team, Inria Sophia Antipolis Méditerranée, Valbonne, France

Université Côte d'Azur, France

E-mail: elif.koksal@inria.fr

present address: INSERM, U1099, LTSI, Campus de Beaulieu, Rennes, France

M. Desroches

MathNeuro Team, Inria Sophia Antipolis Méditerranée, Valbonne, France

Université Côte d'Azur, France

A. Guillamon

Universitat Politècnica de Catalunya, Departament de Matemàtiques, Barcelona, Spain

J. Rinzel

Center for Neural Science, New York University, New York, USA

Courant Institute for Mathematical Sciences, New York University, New York, USA

J. Tabak

University of Exeter Medical School, University of Exeter, Exeter, UK 
give rise mixed-mode bursting oscillations (MMBOs). We unveil complicated isolas along which MMBOs exist in parameter space. The profile of solutions along each isola undergoes canard-mediated transitions between the subthreshold regime and the bursting regime; these explosive transitions change the number of oscillations in each regime. Finally, we relate the MMBO dynamics to experimental recordings and discuss their effects on the silent phases of bursting patterns as well as their potential role in creating subthreshold fluctuations that are often interpreted as noise. The mathematical framework used in this paper is relevant for modelling multiple timescale dynamics in excitable systems.

Keywords rate models, excitability, multiple timescale systems, mixed-mode bursting oscillations, canard solutions.

Mathematics Subject Classification (2010) 34N05

\section{Introduction}

Complex oscillatory patterns arising in Neuroscience (excitability, spiking, bursting and subthreshold oscillations) have been intensively studied via mathematical modelling at multiple spatial and temporal scales, ranging from single cell $[34,24]$ to network level $[41,39]$. These complex dynamics occur due to strong interaction of system variables across various timescales. Multiple timescale (so-called slow-fast or singularly perturbed) dynamical systems provide an efficient ground to study those patterns $[15,19]$. At the network level, the spontaneous episodic activity in developing spinal cord of the chick embryo analysed in $[16,30,31,41,40,39]$ is a clear experimental manifestation of a rich diversity of temporal dynamics. Here, we study the dynamical features of this episodic activity by means of the firing rate model proposed in [41] that accounts for the activity of an excitatory population endowed with fast and slow synaptic depression mechanisms and a slow threshold dynamics.

In slow-fast dynamical systems, the timescale separation parameter $\varepsilon$ is a small scalar quantity $(0<\varepsilon \ll 1)$ that measures the speed difference between the state variables. The presence of $\varepsilon$ results in having distinct groups of slow and fast variables, and the overall dynamical behaviour of the system can be divided into slow and fast epochs. In its most general form, a slow-fast system can be written as follows:

$$
\begin{aligned}
\mathbf{x}^{\prime} & =\mathbf{f}(\mathbf{x}, \mathbf{y}, \varepsilon), \\
\mathbf{y}^{\prime} & =\varepsilon \mathbf{g}(\mathbf{x}, \mathbf{y}, \varepsilon),
\end{aligned}
$$

where $\mathbf{x} \in \mathbb{R}^{n}$ is the vector of fast variables, $\mathbf{y} \in \mathbb{R}^{m}$ is the vector of slow variables and the prime denotes differentiation with respect to the fast time $\tau$; the functions $\mathbf{f}$ and $\mathbf{g}$ are assumed to be sufficiently smooth. Depending on the relative dimensions of slow and fast variables, one can observe different types of complex oscillations such as relaxation oscillations, bursting oscillations, mixed-mode oscillations (MMOs) and mixed-mode bursting oscillations (MMBOs), which are a combination of the previous two; see Figure 1 for examples 
of each of these types of slow-fast oscillations. These complex oscillations are solutions of slow-fast systems of the type (1), periodic or not, which consist of successions of slow small-amplitude and fast large-amplitude oscillations. Geometric singular perturbation theory (GSPT) is a key tool to understand the role of the timescale separation in a given system, the geometry of the problem and the possibility for complex oscillatory dynamics to arise in this system. In this context, canard solutions are important building blocks that organise these complex oscillations in parameter space.

MMBOs found in the model for the episodic activity in the developing spinal cord are the main focus of the present article. Our treatment of this case study serves to illustrate the dynamical features and mechanisms that can be expected in other systems. Episodic activity is composed of active phases of network spikes, so-called episodes, followed by quiescent periods. According to [40], network noise is prominent during quiescence and hence leads to predominantly stochastic transitions from silent phases to active phases. In contrast, the opposite transitions, from active phases to silent phases, are predominantly deterministic. In this paper, we consider the modelling framework of multiple-timescale dynamical systems, whereby fluctuations in the activity before the beginning of an episode naturally come as slow-fast subthreshold oscillations. Although these subthreshold oscillations might not be observable in extracellular electrophysiological recordings, disentangling what pertains to noise from what could correspond to subthreshold oscillatory processes might be very hard. Yet, we show that deterministic subthreshold oscillations might be responsible for delaying the active phase onset sufficiently to increase silent phase duration. Furthermore, we show that MMBOs are organised in parameter space along closed curves typically referred to as isolas. Isolas are bifurcation branches that are isolated in parameter space, that is, they are not directly connected to other branches. They are often found in slow-fast dynamical systems displaying parameterised families of complex oscillations, either MMOs [11] or bursting [43]. Typically, many such isolas coexist and, along each of them, the overall oscillatory profile does not vary, however small oscillations can expand or shrink during canard-explosive segments of the isola (see Figure 11 for an example). The dynamical role of these objects, as well as their behaviour in the singular limit, has not yet been fully unraveled, even though they seem to form a trademark of complex oscillatory dynamics with multiple timescales. Below, we review the basics of canard dynamics in slow-fast systems pertinent to the present study and introduce the model.

\subsection{Canard dynamics in slow-fast systems}

Setting $\varepsilon=0$ in the general form of a slow-fast system given in (1) freezes the dynamics of the slow variables in $\mathbf{y}$ and gives the fast subsystem (so-called layer problem), which describes the dynamics of the fast variables in $\mathbf{x}$ for fixed values of the slow variables (now parameters) and, hence, approximates the dynamics of the original system during fast epochs. 
(a)

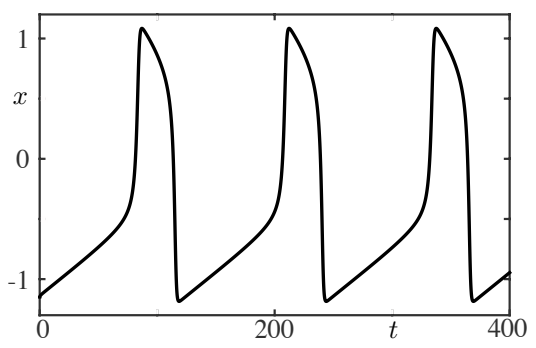

(c)

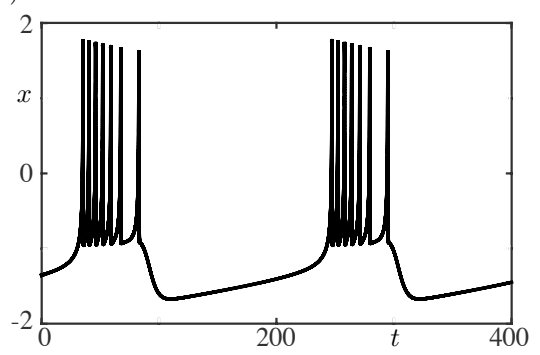

(b)

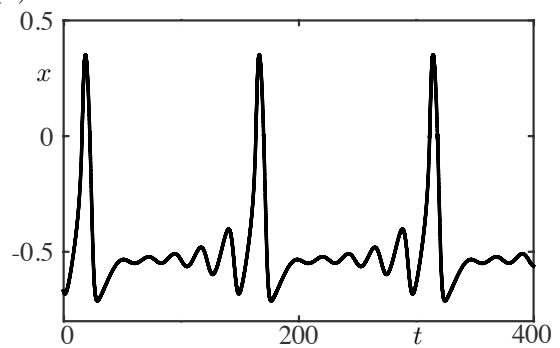

(d)

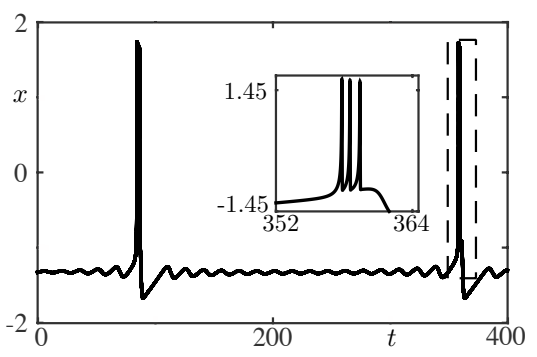

Fig. 1 Example traces of different types of complex oscillations. (a) Relaxation oscillations in a 2-dimensional system of 1 slow and 1 fast variables. During the fast (slow) epochs, the amplitude of $x(t)$ varies sharply (moderately). (b) MMOs in a 3-dimensional system of 2 slow and 1 fast variables. Sharp variations in the amplitude of $x(t)$, hence spikes, appear during the fast epochs, whereas small-amplitude oscillations appear during the slow epochs. (c) Busting oscillations in a 3-dimensional system of 1 slow and 2 fast variables. During the fast epochs $x(t)$ undergoes spikes, whereas during the slow epochs the amplitude of $x(t)$ changes moderately. (d) MMBOs in a 4-dimensional system of 2 slow and 2 fast variables where the inset zooms the bursting activity of the solution marked by the dashed rectangle. During the fast epochs $x(t)$ undergoes spikes, whereas small-amplitude oscillations appear during the slow epochs.

Applying the time rescaling $t=\varepsilon \tau$ gives the slow-time parametrisation of the original system, which reads:

$$
\begin{aligned}
\varepsilon \dot{\mathbf{x}} & =\mathbf{f}(\mathbf{x}, \mathbf{y}, \varepsilon), \\
\dot{\mathbf{y}} & =\mathbf{g}(\mathbf{x}, \mathbf{y}, \varepsilon) .
\end{aligned}
$$

Taking $\varepsilon=0$ in (2) yields the slow subsystem (so-called reduced system), which is a set of differential equations on the slow variables $\mathbf{y}$ constrained by the algebraic condition $\mathbf{0}=\mathbf{f}(\mathbf{x}, \mathbf{y}, 0)$. This algebraic equation defines the critical manifold $S^{0}:=\{\mathbf{f}=\mathbf{0}\}$, which then corresponds to both the phase space of the slow subsystem and to the set of the equilibria of the layer problem.

The stability of the layer problem's equilibria determines whether subsets of the critical manifold can be said to be attracting, repelling or of saddle type. More generally, hyperbolic equilibria of the layer problem correspond by definition to so-called normally hyperbolic points of $S^{0}$. This means that parts of the critical manifold, where the dynamics in the normal direction 
is hyperbolic, perturb smoothly in $\varepsilon$ to give so-called slow manifolds of the original system for small enough $\varepsilon>0$. Indeed, Fenichel theory [17] guarantees the existence of locally invariant (attracting, repelling or saddle-type) slow manifolds $S^{\varepsilon}$ as perturbations of compact normally hyperbolic (attracting, repelling or saddle-type) submanifolds of $S^{0}$.

Attracting and repelling branches of $S^{0}$ ( $S^{a}$ and $S^{r}$, respectively) meet along the fold set

$$
F:=\left\{\left(\mathbf{x}_{\text {fold }}, \mathbf{y}_{\text {fold }}\right) \in S^{0} ;\left(D_{\mathbf{x}} \mathbf{f}\left(\mathbf{x}_{\text {fold }}, \mathbf{y}_{\text {fold }}, 0\right)\right)=\mathbf{0}\right\}
$$

which corresponds to a saddle-node bifurcation set of the layer problem. Since normal hyperbolicity is lost at every point of $F$, Fenichel theory does not apply and different mathematical techniques must be employed in order to understand the dynamics of the original system near $F$. The usual strategy is to analyse the slow flow (2) in the singular limit $\varepsilon=0$ by differentiating $S^{0}$ with respect to time, giving

$$
\begin{aligned}
-\operatorname{det}\left(D_{\mathbf{x}} \mathbf{f}\right) \dot{\mathbf{x}} & =\left(\operatorname{adj}\left(D_{\mathbf{x}} \mathbf{f}\right) \cdot D_{\mathbf{y}} \mathbf{f} \cdot \mathbf{g}\right)(\mathbf{x}, \mathbf{y}, 0), \\
\dot{\mathbf{y}} & =\mathbf{g}(\mathbf{x}, \mathbf{y}, 0),
\end{aligned}
$$

where $\operatorname{adj}\left(D_{\mathbf{x}} \mathbf{f}\right)$ denotes the adjugate of the matrix $D_{\mathbf{x}} \mathbf{f}$. This system is singular on the fold points when $\operatorname{det}\left(D_{\mathbf{x}} \mathbf{f}\right)=0$. To overcome the singularity problem, one can use a rescaling factor $\operatorname{det}\left(D_{\mathbf{x}} \mathbf{f}\right)$ and obtain the so-called desingularized reduced system (DRS), whose equations take the form:

$$
\begin{aligned}
& \mathbf{x}^{\prime}=\left(\operatorname{adj}\left(D_{\mathbf{x}} \mathbf{f}\right) \cdot D_{\mathbf{y}} \mathbf{f} \cdot \mathbf{g}\right)(\mathbf{x}, \mathbf{y}, 0), \\
& \mathbf{y}^{\prime}=-\operatorname{det}\left(D_{\mathbf{x}} \mathbf{f}\right) \mathbf{g}(\mathbf{x}, \mathbf{y}, 0)
\end{aligned}
$$

Equilibria of the DRS satisfying $\operatorname{det}\left(D_{\mathbf{x}} \mathbf{f}\right)=0$ are called folded equilibria or folded singularities of the original system. Hence, saddle, node and saddlenode equilibria of the DRS give folded-saddle, folded-node and folded-saddlenode singularities, respectively. Folded singularities are dynamically interesting objects mainly because they are known to give rise to canard solutions. A canard is a solution of a slow-fast system which flows successively near $S^{a}$ towards $S^{r}$, by passing close to a folded singularity, which in planar systems is called a canard point $[3,25]$. Note that folded foci do exist but are much less interesting as they do not give rise to canards. For detailed descriptions of folded singularities, see e.g. [38] and [44].

\subsection{Canards in neuronal models}

Canards are special solutions of slow-fast dynamical systems which enjoy the counter-intuitive property of staying close, during long time-intervals, to regions of phase space where the flow is highly repulsive. Initially discovered in the context of the van der Pol oscillator (VDP), hence naturally present in the FitzHugh-Nagumo neuron model, they can be shown to occur in a large class of excitable systems, in particular in type-II neuron models such 

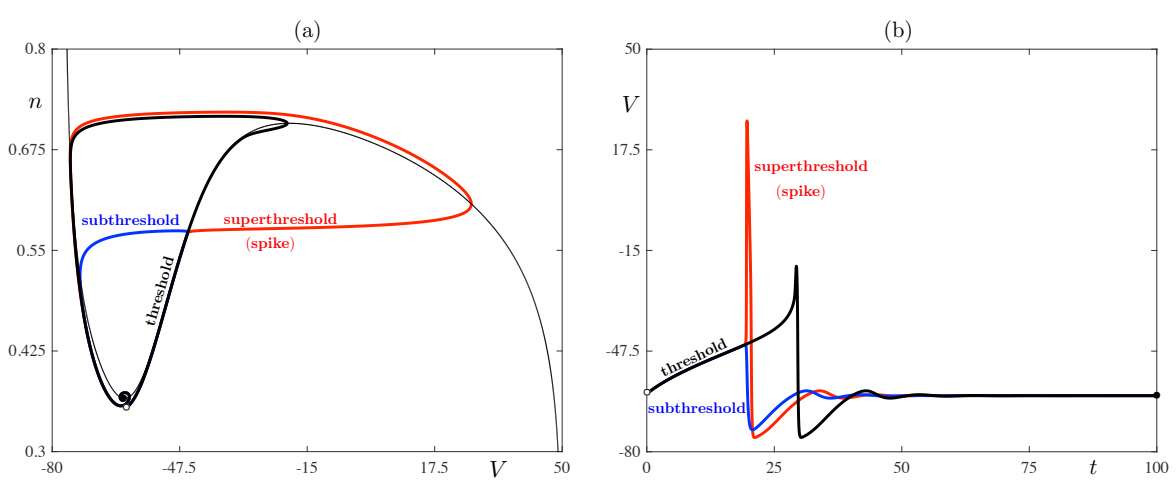

Fig. 2 Canards and excitability in a nutshell in a 2D reduction of the Hodgkin-Huxley model [28]. (a) $(V, n)$-phase plane projection, where $V$ and $n$ represent the membrane potential and sodium channel dynamics, respectively. The cubic-shaped curve is the fast nullcline or the critical manifold of the system. We consider a parameter set in the excitable regime given in [28], i.e. with one stable equilibrium (black dot). We take the same initial condition (white circle) and three different values of the applied current $I$ within such small range than the different $I$-values agree up to the 10th decimal place. However, the solution profiles vary enormously. Even though all of them eventually go to the rest state, one trajectory (in blue) stays subthreshold, one (in black) slowly follows the threshold for the longest possible time, and the last one (in red) goes over threshold and emits a spike before going back to the rest state. All three trajectory contain a canard "near-threshold" segment, and the black one contain the longest or maximal canard segment, it provides the best approximation to the actual firing threshold. (b) Membrane potential time profile of the three trajectories in (a).

as the FitzHugh-Nagumo, various reductions of the Hodgkin-Huxley or the Morris-Lecar models ${ }^{1}$. In non-mathematical terms, these systems possess a hysteresis loop acting on the fast modes and involving two families of stable states, namely up and down states, which can be dynamically visited in alternation upon a slow variation of the associated control parameter, describing firing and subthreshold activity, respectively. This alternation introduces the concept of excitability threshold that is familiar to biologists and physicists as a boundary - a priori not observable - governing the all-or-none response of excitable systems like neurons (or lasers) to an external perturbation such as an applied current (or a light beam). The key highlight of canards' role in such excitable systems, which are dynamically (locally or globally) similar to type-II neuron models, is that they precisely materialise these phase-space boundaries between very different activity regimes, in particular between resting and spiking states in the neuronal context. To this extent, a "failed spike" (blue solution in Fig. 2) or a "delayed spike initiation" (red solution in Fig. 2) can be thought of as experimental evidences of the presence of canards in an appropriate underlying neuron model. In larger-dimensional systems, the role of canards extends to form phase-space boundaries between oscillatory

\footnotetext{
${ }^{1}$ Neurons are said to have type-II excitability when they cannot fire action potentials below a positive minimal frequency; from the modelling standpoint they correspond to systems operating near a Hopf bifurcation [15].
} 
states with multiple amplitudes and frequencies. Hence they control the time profile of oscillatory states, which may act as information encoders. Therefore, canards should be considered as building blocks of excitability as well as modulators of information in a large class of models.

From the mathematical standpoint, the sharp transition between equilibria and relaxation cycles is manifested by an abrupt increase in the cycles' amplitude (referred to as a canard explosion) that occurs within an exponentially small (in $\varepsilon$ ) parameter range and it is in particular observed in the VDP system, which can be seen as a caricature of a type-II excitable neuron. In the VDP type systems, the critical manifold is one-dimensional and S-shaped: it has two attracting branches separated by a repelling branch. In such systems, canards without head solutions correspond to limit cycles with segments on one of two attracting branches of the critical manifold and on the repelling branch (blue trajectory in Fig. 2); on the other hand, canard with head solutions correspond to limit cycles with segments on two attracting branches of the critical manifold and on the repelling branch (red trajectory in Fig. 2). These two families of canard cycles are connected in parameter space by the so-called maximal canard, which by definition is the limit cycle solution with the longest repelling segment (black trajectory in Fig. 2). In the neuronal context, the maximal canard provides the best approximation of the excitability threshold for type-II neurons and hence stands as a clear boundary between subthreshold oscillations (corresponding to canards without head) and spiking solutions (corresponding to canards with head and then to relaxation cycles) $[10,13,28]$. The role of canards in the synchronisation of $2 \mathrm{D}$ excitable systems has recently started to be unveiled [21,22].

In three-dimensional (3D) slow-fast systems, canards play a similar crucial role in approximating firing thresholds $[27,45]$ and in organising complex oscillations, such as MMOs and bursting oscillations. MMO-type solutions combine small-amplitude oscillations (SAOs) and large-amplitude oscillations (LAOs) of relaxation type, in a recurrent (periodic or not) fashion (Fig. 1-(b)). They arise in 1 fast/ 2 slow systems possessing a folded-node or a folded-saddlenode singularity. Such MMO systems can be interpreted as canard oscillators (of VDP type) with a drift that allows to flow through the canard point of the associated canard oscillator, and a global reinjection mechanism ensuring that these dynamics recur [7,44]; see the review article [11] for details. In neuronal models, the appearance of subthreshold oscillations and subsequent transitions to spikes can be governed by an underlying canard structure $[20,24,36,37]$.

Bursting oscillations can appear in 3D slow-fast systems with 2 fast and 1 slow variables, and they correspond to fast oscillations (that is, in the neuronal context, a sequence of spikes) followed by a quiescent phase during which the system is slowly evolving (Fig. 1-(c)). Most models of bursting that have been characterized correspond to 2 fast/1 slow systems and their $2 \mathrm{D}$ fast subsystems are bistable oscillators where the bistability is typically between limit cycles and equilibria.

The classical interpretation of bursting oscillations as recurrent slow-fast transitions between quiescence and spiking is due to Rinzel $[33,35,34]$, who 
had the seminal idea to relate such transitions to bifurcations of the fast subsystem. Based on this idea, Rinzel came up with a classification of bursting patterns that include three classes (square-wave, elliptic, parabolic). As proposed by Izhikevich in [19], one can further classify many types of bursting oscillations by identifying in each class two bifurcations of the fast subsystem, one starting the bursting and one ending it. Soon after Rinzel's classification was proposed, Terman identified a spike-adding phenomenon in a square-wave burster built upon the Morris-Lecar spiking model with an additional slow variable [42]. This slow-fast phenomenon involves canard trajectories organising spike-adding transitions and saddle-type slow manifolds; since then it has been found in a number of bursting models [8,12,14,18,29,32,43,23]. These studies also revealed that canards play a similar role in the transition from rest to bursting in (at least) 3D slow-fast systems as they do in the transition from rest to spiking in planar systems.

MMO and bursting dynamics can be combined within the framework of four-dimensional (4D) systems with 2 slow and 2 fast variables resulting in complex oscillatory dynamics of the type MMBOs [12] (Fig. 1-(d)). MMBOtype complex oscillations arise due to a slow passage through a spike-adding canard explosion in certain types of bursting systems where the burst is initiated through a saddle-node bifurcation of the fast subsystem. One distinct feature of these canard-mediated MMBOs which distinguishes them from elliptic bursting's silent phase oscillations is that they involve two slow variables oscillating together whereas in the elliptic bursting case these oscillations are solely due to the presence of focus equilibria in the fast subsystem, hence only one slow variable is involved. Desroches et al. [12] described MMBOs in an extended version of the Hindmarsh-Rose system with a slowly-varying external input. They also discussed the presence of these dynamics in the Morris-LecarTerman system [42] and a biological modelling of secreting neurons [5].

\subsection{The model}

Following experimental observations [16,30,31], Tabak et al. have modelled the network activity using a rate model formalism with activity-dependent network depression and the assumption of excitatory connectivity between underlying neurons. To this end, they introduced two fast $(a, d)$ and two slow $(s, \theta)$ variables, which represent the activity (population activity or mean firing rate) (variable $a$ ), the fraction of synapses not affected by the fast synaptic depression $(d)$, the fraction of synapses not affected by the slow synaptic depression $(s)$ and the threshold for the cell firing $\theta$, respectively. The resulting $4 \mathrm{D}$ system reads:

$$
\begin{aligned}
\tau_{a} \dot{a} & =a_{\infty}\left(w d s a-\theta-\theta_{0}\right)-a, \\
\tau_{d} \dot{d} & =d_{\infty}(a)-d, \\
\tau_{\theta} \dot{\theta} & =\theta_{\infty}(a)-\theta, \\
\tau_{s} \dot{s} & =s_{\infty}(a)-s,
\end{aligned}
$$


with sigmoid functions:

$$
\begin{aligned}
& a_{\infty}(v)=1 /\left(1+\mathrm{e}^{-(v) / k_{a}}\right), \\
& d_{\infty}(a)=1 /\left(1+\mathrm{e}^{\left(a-\theta_{d}\right) / k_{d}}\right), \\
& \theta_{\infty}(a)=1 /\left(1+\mathrm{e}^{-\left(a-\theta_{\theta}\right) / k_{\theta}}\right), \\
& s_{\infty}(a)=1 /\left(1+\mathrm{e}^{\left(a-\theta_{s}\right) / k_{s}}\right) .
\end{aligned}
$$

Tabak et al. considered various 3D subsystems of (4) in order to study different aspects of the dynamics. The $(a, d, \theta)$-model (where $s$ does not appear in the $a$-equation) and the $(a, d, s)$-model (where $\theta$ is a parameter) reproduce the experimentally observed bursting behaviour [41]: the slow variables $\theta$ (in the $(a, d, \theta)$-model) and $s$ (in the $(a, d, s)$-model) control the periodic transitions between the active (fast oscillations due to the interaction between $a$ and $d)$ and the silent phase. On the other hand, the $(a, \theta, s)$-model [39] does not account for the fast synaptic kinetics but instead captures the impact of the cellular excitability and the network connectivity on the durations of the active and silent phases of the episodic activity. The resulting behavior of the $(a, \theta, s)$ model is a relaxation cycle in the $3 \mathrm{D}$ phase space.

Although these 3D reductions help to understand the effects of underlying biological variables on the network activity, they both produce a ratio of silent and active phase durations much smaller than experimentally observed. Here we show that subthreshold oscillations before the beginning of an episode could lengthen the silent phase and thus produce a higher ratio of silent to active phase duration. Figure 3 shows an example of these subthreshold oscillations followed by a burst, hence forming a MMBO solution, obtained by simulating the $(a, d, \theta, s)$-model (4). Complex oscillations of this type might be responsible for delaying the active phase onset sufficiently to increase silent phase duration and the aim of the present study is to analyse them, both theoretically and numerically, in system (4). To do so, we focus on the canard structures and associated canard-mediated dynamics in the $4 \mathrm{D}$ rate model (4) as well as in its two aforementioned reductions, and their impact on the complex oscillatory activity of the network. In Section 2, we detail the canard-mediated spikeadding transitions in the bursting regime of the $(a, d, \theta)$-model. In Section 3, we unveil and analyse MMO dynamics in the $(a, \theta, s)$-model. Then, in Section 4 , we explain how to combine the bursting dynamics of the $(a, d, \theta)$-model and the MMO dynamics of the $(a, \theta, s)$-model into MMBOs using the $(a, d, \theta, s)$ model. Finally, in Section 5, we summarise our findings, their relevance to the neural system under investigation and propose further avenues of research in this direction. Throughout the paper, we shall use the parameter set given in [41], also provided in the caption of Figure 3, leaving the network connectivity measure $w$ as the main bifurcation parameter; see [41] for detailed description of the parameters. 


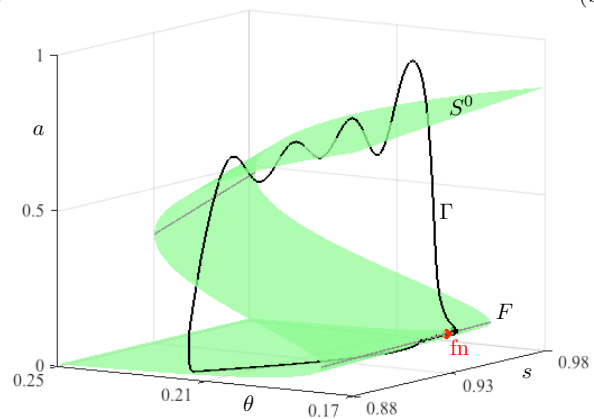

(b)

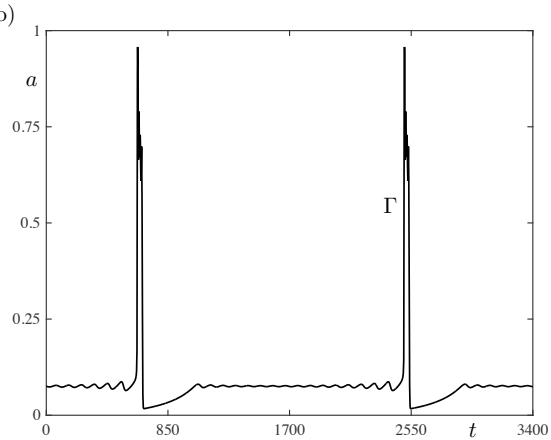

Fig. 3 System (4) with $w=1.43$ and $\tau_{a}=1, k_{a}=0.05, \tau_{d}=2, \theta_{d}=0.2, k_{d}=0.5$, $\tau_{\theta}=1000, \theta_{\theta}=0.15, k_{\theta}=0.05, \tau_{s}=500, \theta_{s}=0.14, k_{s}=0.02, \theta_{0}=0$. (a) MMBO solution $\Gamma$ projected onto the $(\theta, s, a)$-space. Also shown are the critical manifold $S^{0}$, the fold curve $F$ and the folded node fn (red dot). (b) Time profile of $\Gamma$ for the $a$-variable.

\section{Canard-mediated transitions to burst and spike-adding in the $(a, d, \theta)$-model}

Tabak et al. considered two 3D reductions of the $(a, d, \theta, s)$-model with 2 fast $/ 1$ slow variables, the $(a, d, \theta)$ and $(a, d, s)$ models [41]. In both systems, the fast variables, $a$ and $d$, form a bistable system of oscillations and equilibria parameterised by the cellular threshold $\theta$ and the activity-dependent depression $s$, respectively. Slow modulations in $\theta$ in the $(a, d, \theta)$-model and $s$ in the $(a, d, s)$ model govern recurrent dynamic transitions in the full 3D system between the oscillatory and stationary states of the $(a, d)$ fast subsystem, which result in the bursting behaviour.

Canard-induced dynamics in bursting systems has been unveiled in several models $[12,14,18,29,32,42,43]$, where bursting is initiated, upon parameter variation, via a Hopf bifurcation followed by a sequence of canard explosions and spikes are shaped via canard trajectories. In this section we investigate canard-induced dynamics in the $(a, d, \theta)$-model keeping $s$ as the bifurcation parameter. We use the same parameter set for which MMBO solutions exists in (4) (Figure 3 and Section 4), but change $\tau_{\theta}=5000$ for a clear visualisation of transitions. Note that similar study in the $(a, d, s)$-model with the parameter $\theta$ will give qualitatively similar slow-fast transitions.

The $(a, d, \theta)$-model reads:

$$
\begin{aligned}
\tau_{a} \dot{a} & =a_{\infty}\left(w d s a-\theta-\theta_{0}\right)-a, \\
\tau_{d} \dot{d} & =d_{\infty}(a)-d, \\
\dot{\theta} & =\varepsilon\left(\theta_{\infty}(a)-\theta\right),
\end{aligned}
$$

where $\varepsilon=1 / \tau_{\theta}$ being the small parameter (given that $\tau_{a}=1$ and $\tau_{d}=2$ ). System $(6)$ undergoes a supercritical Hopf bifurcation at $s_{H o p f} \approx 0.95657$. As in the canard explosion scenario in planar systems, the canard solutions of 
(6) lie along a quasi-vertical segment of the solution branch in the bifurcation diagram in terms of $s$. This quasi-vertical segment is located at an $O\left(1 / \tau_{\theta}\right)$ distance of the Hopf point at $s_{H o p f}$, that is, near the canard value at $s_{c} \approx$ 0.95704. Next, we explain how canard orbits are formed from the early stages of the canard explosion until the formation of the first spike (Figure 5), as well as for the second and third spikes (Figures 6).

In order to understand the canard trajectories structuring these periodic orbits, we need to examine the bifurcation diagram of the $(a, d)$ fast subsystem with respect to $\theta$ for $s \approx s_{c}$. The $\theta$-dependent family of equilibrium points $\left(a^{*}, d^{*}\right)=\left(a^{*}, d_{\infty}\left(a^{*}\right)\right)$ of the fast subsystem is defined by

$$
\left\{a_{\infty}\left(w d s a-\theta-\theta_{0}\right)-a=0\right\} \cap\left\{d_{\infty}(a)-d=0\right\}
$$

that is,

$$
\left\{\theta=k_{a} \ln \left(\frac{1}{a}-1\right)+w d_{\infty}(a) s a-\theta_{0}\right\},
$$

and forms a Z-shaped curve for $a \in(0,1)$ (blue curve in Figure 4) with two fold points, $\theta_{f}^{l}$ and $\theta_{f}^{r}$. The lower branch consists of stable nodes for $\theta>\theta_{f}^{l}$. Saddle equilibria, whose invariant manifolds are weaker in the stable direction than in the unstable direction, lie along the middle branch between $\theta_{f}^{l}$ and $\theta_{f}^{r}$. The upper branch has stable foci until the supercritical Hopf bifurcation at $\theta_{H}$, which gives a narrow paraboloid of limit cycles. This paraboloid folds back at $\theta_{F C}$ towards the middle branch of saddles and makes a saddle-homoclinic connection at $\theta_{H C}$. The bursting behaviour resulting from this bifurcation structure in the $(a, d)$ fast subsystem is classified as a "Hopf/ fold cycle" bursting by Izhikevich [19]. The homoclinic connection at $\theta_{H C}$ is one of the main ingredients of the spike adding phenomena explained below.

After having dissected the $(a, d)$ subsystem with respect to $\theta$, we can focus on the slow-fast transitions in (6). Equation (7) defines the critical manifold $S^{0,(a, d, \theta)}$ of $(6)$ where the only slow variable is $\theta$. Therefore, the $1 \mathrm{D}$ slow flow lies along the folded set:

$$
F^{(a, d, \theta)}=\left\{\frac{-k_{a}}{a(1-a)}+w s\left(a d_{\infty}^{\prime}(a)+d_{\infty}(a)\right)=0\right\}
$$

with $d_{\infty}^{\prime}(a)=\frac{d}{d a}\left(d_{\infty}(a)\right)=-\frac{e^{\left(a-\theta_{d}\right) / k_{d}}}{k_{d}\left(1+e^{\left(a-\theta_{d}\right) / k_{d}}\right)^{2}}$. The critical points of $S^{0,(a, d, \theta)}$, i.e. the fold points, are at $\theta_{f}^{l}$ and $\theta_{f}^{r}$. zoThe passage of the slow nullcline $\left\{\theta=\theta_{\infty}(a)\right\}$ through the fold point $\theta_{f}^{l}$ in the 3D phase space as $s$ slowly varies gives the transition from resting to spiking, then adds the spikes successively via canard trajectories until the bursting regime is reached.

The upper panels of Figure 5 show the first canard cycles induced by the Hopf bifurcation at $s_{\text {Hopf }}$ in a similar analogy to that of the VDP-type planar systems: the trajectories move along the lower branch of stable nodes, pass through $\theta_{f}^{l}$ and follow $1 \mathrm{D}$ repelling slow manifolds before jumping to 
(a)

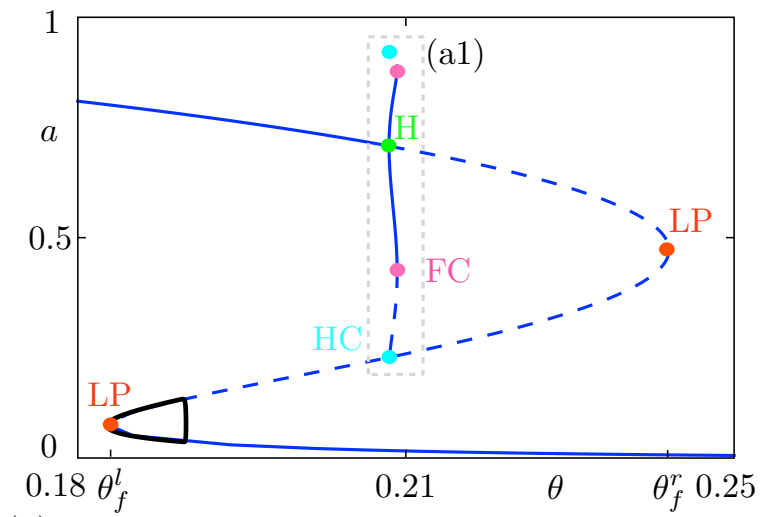

(a1)

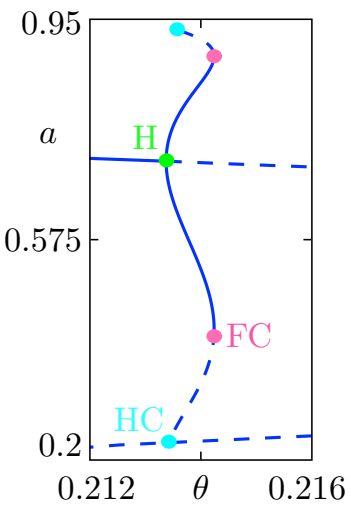

(b)

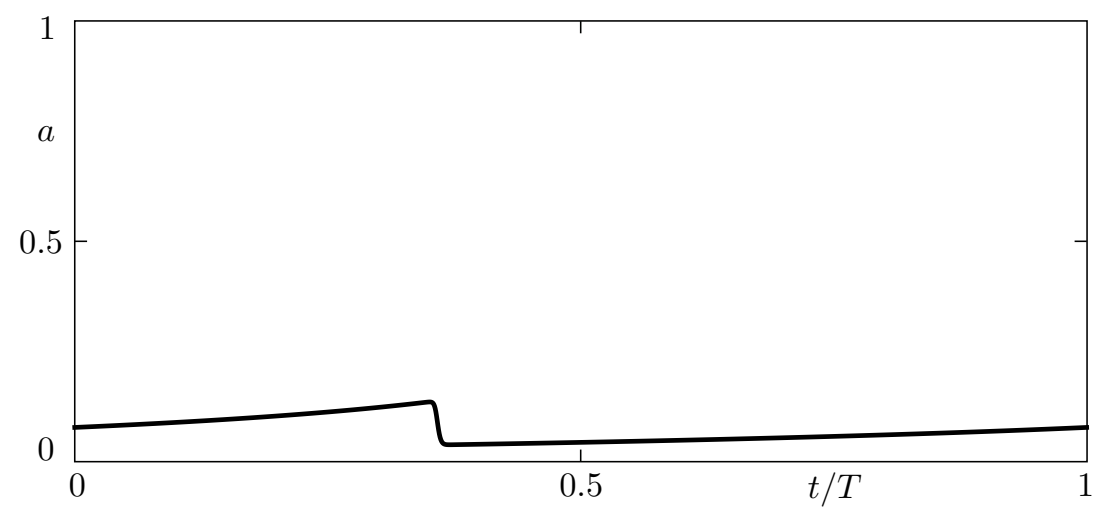

Fig. 4 (a) Bifurcation diagram of the $(a, d)$ fast subsystem upon variation of $\theta$ (blue), together with an example canard cycle of the $(a, d, \theta)$ system for $s \approx s_{c}$ (black). Panel (a1) corresponds to a zoom of panel (a) in the dashed rectangle. In both upper panels, $\mathrm{H}$ denotes a Hopf bifurcation, LP limit point bifurcations (folds) of equilibria, HC a Homoclinic bifurcation and FC a fold bifurcation of cycles. The equilibrium points on the Z-shaped (blue) curve are stable nodes along the lower branch for $\theta>\theta_{f}^{l}$ and saddles between the fold bifurcation values $\theta_{f}^{l}=0.18326$ and $\theta_{f}^{r}=0.2432$. The stable focus type equilibria along the upper branch undergo a supercritical Hopf bifurcation at $\theta_{H} \approx 0.21335$, which gives a narrow paraboloid of limit cycles. The inner cycles, between $\theta_{H}$ and $\theta_{F C} \approx 0.21423$, are stable, while the outer cycles, between $\theta_{F C}$ and $\theta_{H C} \approx 0.21343$, are unstable. The upper branch hosts unstable equilibria of focus type between $\theta_{H}$ and $\theta \approx 0.241448$, then unstable nodes until $\theta_{f}^{r}$. (b) Time series for variable $a$ of the limit cycle shown in panel (a).

back to the branch of stable nodes (Fig. 5-(1)). As $s$ varies along the canard explosion, the canard cycles grow in amplitude up to the maximal canard while moving towards $\theta_{f}^{r}$. These spikeless cycles are analogous to the canard without head trajectories in planar systems and the maximal canard is the excitability threshold of the $(a, d, \theta)$-system.

When the limit cycle reaches to the right fold $\theta_{f}^{r}$, the (un)stable manifolds of the equilibria shape the cycle. The invariant set of the saddle at $\theta_{f}^{r}$ 
expands the cycle in the $a$-direction, then brings it back towards the branch of the stable nodes (similar expansion-contraction mechanism is shown in [18, Figure 6]). This expansion and contraction at $\theta_{f}^{r}$ gives birth to the first spike (Fig. 5-(4)). As $s$ varies, the trajectory is repelled in the $a$-direction by the unstable manifolds of saddle points of the middle branch then attracted by the stable lower branch of $S^{0,(a, d, \theta)}$. This behaviour is similar to the canard with head cycles in the planar systems where a trajectory follows both attracting branches of the critical manifold. However, in the $(a, d, \theta)$-system, the critical manifold contains repelling equilibria along the upper branch between $\theta_{f}^{l}$ and $\theta_{H}$. Thus, the trajectory returns to the lower branch once leaves the middle branch.

The formation of a cycle with one spike is completed once the spike is placed on the left of $\theta_{\text {Hop }}$. Note that it is still a canard cycle since a certain part of it lies along the unstable middle branch: it follows the stable branch as $\theta$ decreases towards $\theta_{f}^{l}$, passes through the fold $\theta_{f}^{l}$, continues along the unstable middle branch before departing in the direction of the unstable manifold towards the attracting focus. Since the $(a, d)$ subsystem does not possess any cycle for $\theta<\theta_{H}$ and the stable foci have weak attractions, the trajectory does not necessarily follow the upper branch of equilibria of the fast subsystem.

As $s$ varies, the trajectory can follow again the 1D saddle-type slow manifold provided the trajectory is exponentially close to the repelling branch of the critical manifold. This can only happen in the vicinity of the homoclinic bifurcation of the fast subsystem (for small enough $\varepsilon>0$ ). Hence, as the trajectory comes close to the homoclinic point after the spike, it can grow a new canard segment and this initiates the formation of the next spike [18], see Figure 6 . The second canard segment of the trajectory after the spike expands from $\theta_{H C}$ to $\theta_{f}^{r}$ : the limit cycle with one spike grows in the $\theta$ direction, gains the second spike at the right fold and then shrinks towards the left fold. Different than the first one, the second spike is aligned within the stable limit cycles of the $(a, d)$ subsystem - which gives the fast oscillations of the burst episode. Note that the slow drift in $\theta$ may not allow the second spike to enter into the narrow paraboloid of the stable limit cycles (between $\theta_{H}$ and $\theta_{F C}$ ). As for the third (and later subsequent spikes), the family of stable cycles are drifted "inside the paraboloid" after passing through $\theta_{H}$, remain closer to the stable objects in that region Figure 6-(18). These fast oscillations terminate near $\theta_{F C}$.

\section{Folded node and MMOs in the $(a, \theta, s)$-model}

In this section we investigate how the presence of subthreshold oscillation at the transition between silent and active phases may occur due to the interplay between slow variables $s$ and $\theta$. The oscillations would lengthen the silent phase intervals relative to the active phase duration.

Tabak et al. have studied the effects of both types of slow negative feedback, synaptic depression (s, divisive feedback) and adaptation of cellular excitability 

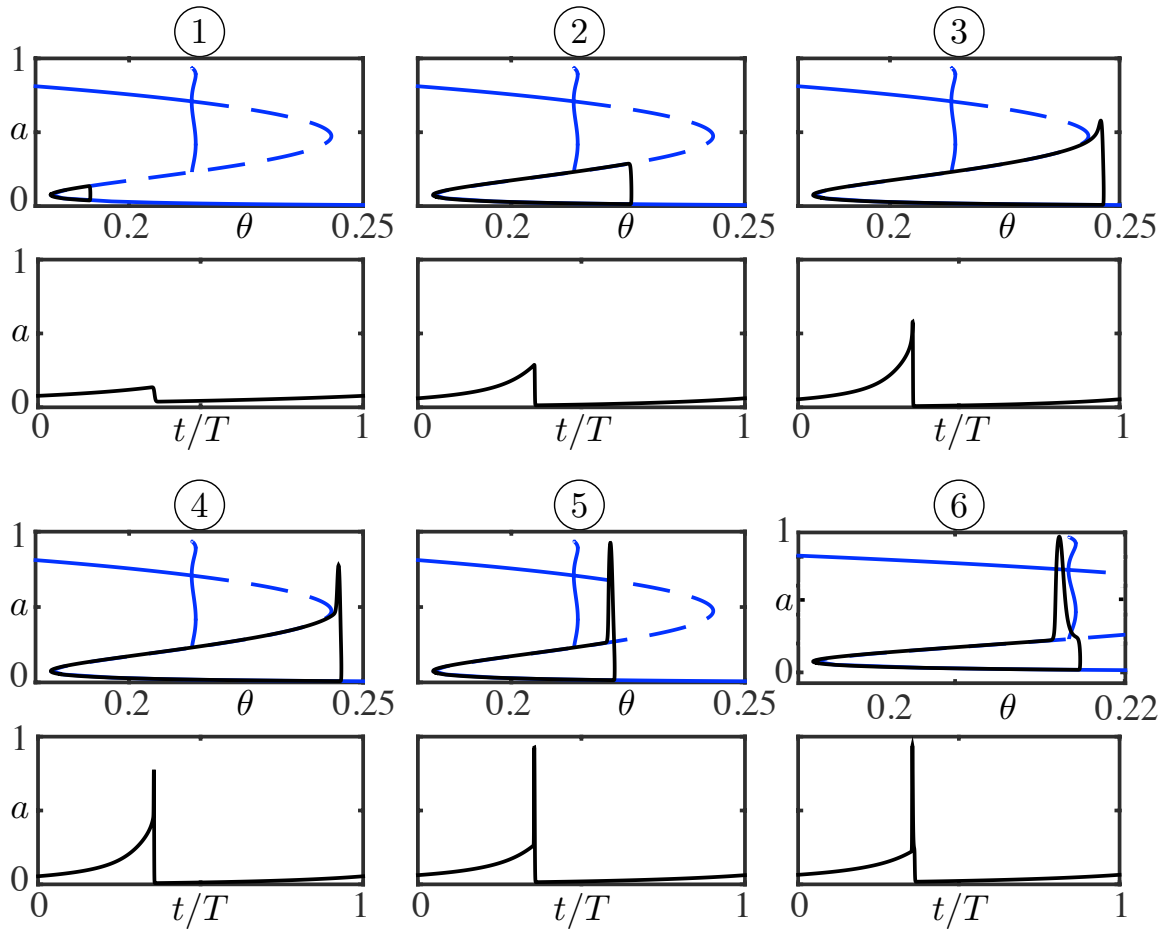

Fig. 5 Formation of the first spike in system (6) upon exponentially small variation of $s \approx s_{c}$. Each up-down pair shows a solution superimposed on the bifurcation diagram of the $(a, d)$ fast subsystem versus $\theta$ (upper panel) and the time course of the solution for variable $a$ (lower panel). Panel (6) displays a zoomed view so as to better visualize the interaction between the spike and the bifurcation diagram of the fast subsystem.

$(\theta$, subtractive feedback), on the active and silent phases of episodic activity via the $(a, \theta, s)$-model [39], which reads

$$
\begin{aligned}
\tau_{a} \dot{a} & =a_{\infty}\left(\tilde{w} s a-\theta-\theta_{0}\right)-a, \\
\dot{\theta} & =\varepsilon\left(\theta_{\infty}(a)-\theta\right), \\
\dot{s} & =\varepsilon \tilde{\tau}_{s}\left(s_{\infty}(a)-s\right),
\end{aligned}
$$

where $\tilde{\tau}_{s}=\tau_{\theta} / \tau_{s}$. The $(a, \theta, s)$-model does not account for the fast kinetics of the activity-dependent synaptic depression, thus its effective network connectivity, $\tilde{w}$, takes different values than $w$ of the $(a, d, \theta, s)$-model. (We use $\tilde{w}$ in the following calculations but the dynamics remain qualitatively the same with a quasi-steady state approximation $d=d_{\infty}(a)\left(\tilde{w} \approx w d_{\infty}(a)\right)$.) The number of slow variables determines the dimension of the critical manifold and, hence, that of the slow flow on it. With the usual parameter set $\left(\tau_{a}=1, \tau_{\theta}=1000, \tilde{\tau}_{s}=\tau_{\theta} / \tau_{s}=2\right)$, the $(a, \theta, s)$-model has one fast $(a)$ and two slow variables $(s, \theta)$, therefore the $a$-nullsurface in (8) defines the critical 

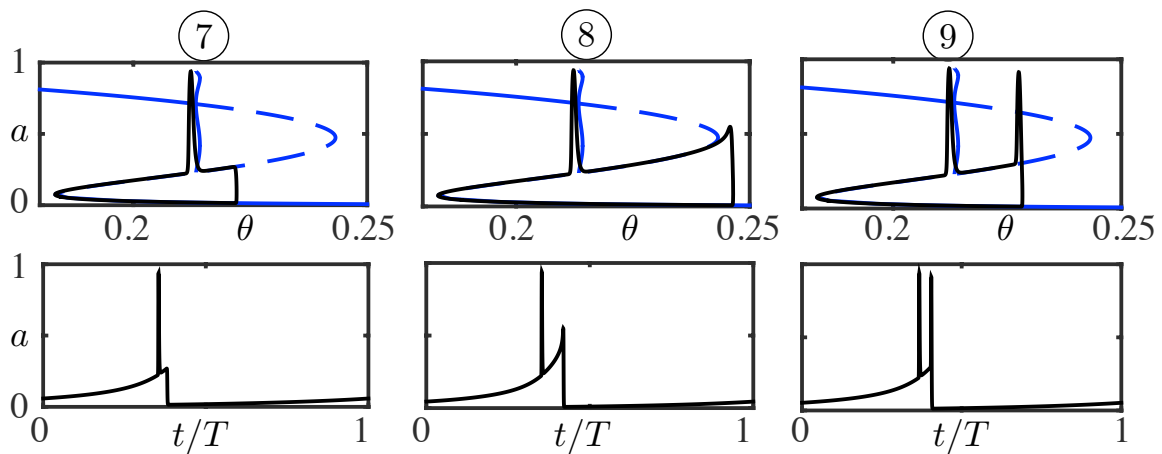

(10)

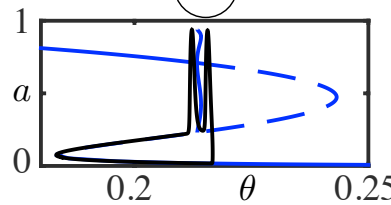

(11)

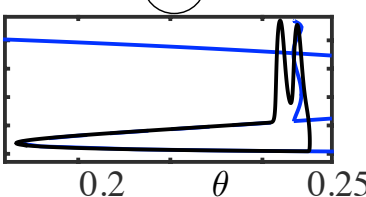

(12)
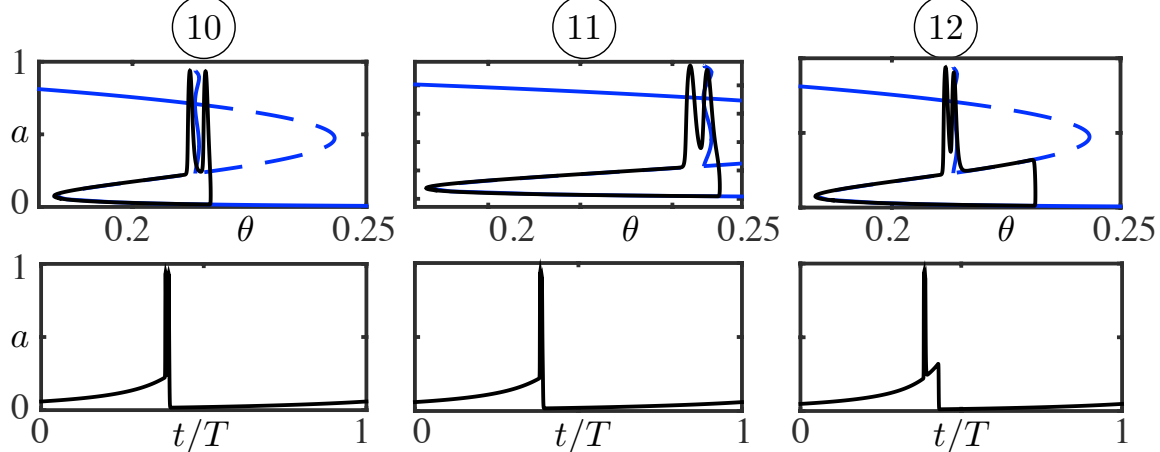

13

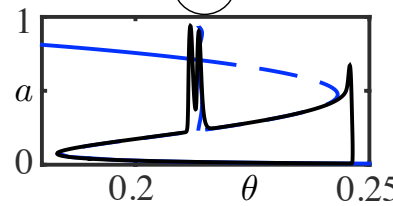

14

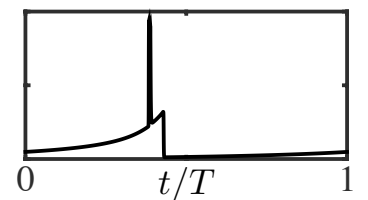

15
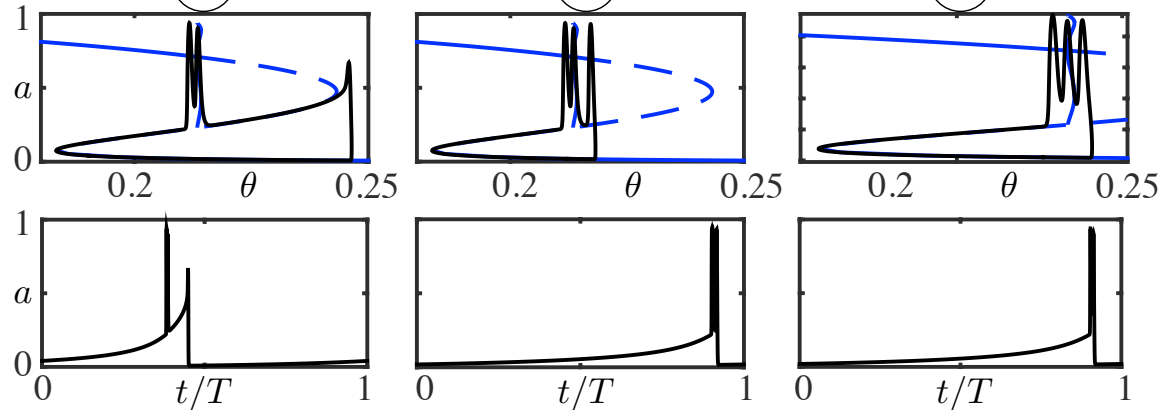

Fig. 6 Formation of the second (panels (7) -(11)) and third (panels (12) -(15)) spikes in system $(6)$ upon exponentially small variations of $s \approx s_{c}$. Each up-down pair shows a solution superimposed on the bifurcation diagram of the $(a, d)$ fast subsystem versus $\theta$ (upper panel) and the time course of the solution for variable $a$ (lower panel). Panels (11) and (15) display zoomed views so as to better visualize the interaction between the spikes and the bifurcation diagram of the fast subsystem.

manifold:

$$
\begin{aligned}
S^{0,(a, \theta, s)} & :=\left\{a_{\infty}\left(\tilde{w} s a-\theta-\theta_{0}\right)-a=0\right\} \\
& :=\left\{k_{a} \ln \left(\frac{1}{a}-1\right)+\tilde{w} s a-\theta-\theta_{0}=0\right\} .
\end{aligned}
$$


(a)

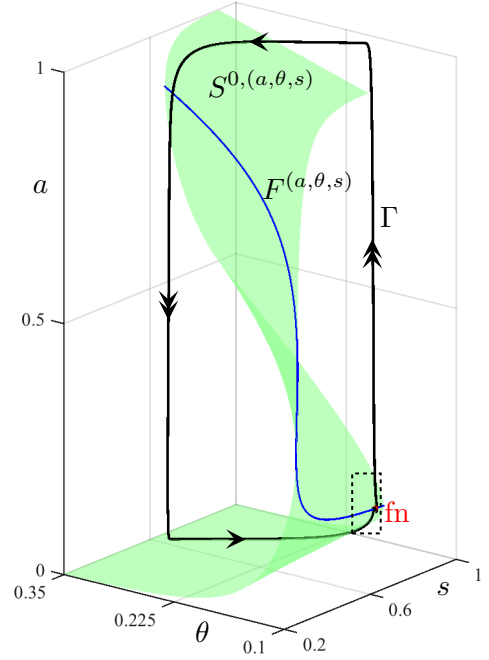

(b)

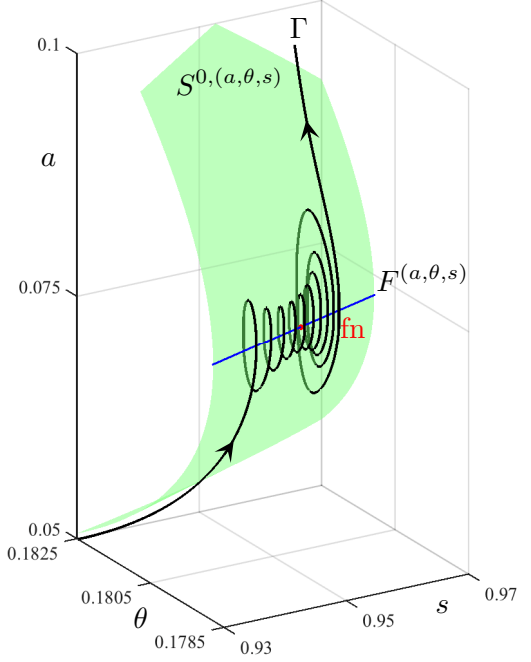

Fig. 7 (a) MMO solution $\Gamma$ of system (8) for $\tilde{w}=0.7625$; also shown are the critical manifold $S^{0,(a, \theta, s)}$, the fold curve $F^{(a, \theta, s)}$ (blue) and the folded node fn (red dot). The critical manifold $S^{0,(a, \theta, s)}$ is a cusp surface folded along the curve $F^{(a, \theta, s)}$. (b) zoom near the folded node (dashed rectangle in panel (a)).

Figure 7 shows $S^{0,(a, \theta, s)}$ with the fold curve (blue), that is

$$
F^{(a, \theta, s)}=\left\{\frac{-k_{a}}{a(1-a)}+\tilde{w} s=0\right\} .
$$

The black curve shown in Figure 7 is an example MMO-type periodic orbit of (8). As we have mentioned in the introduction, the SAOs of MMOs appear due to the presence of folded singularities on the fold curve, in particular near a folded node. Below we investigate the limiting slow dynamics near $F^{(a, \theta, s)}$ using the DRS approach described in the introduction. Note that system (8) is expressed in the fast-time parametrisation. A time rescaling by $\varepsilon=1 / \tau_{\theta}$ brings it to the slow-time formulation, which then allows to compute the DRS. As a first step, we set $\varepsilon$ to 0 in the slow-time system, which gives the following differential-algebraic problem:

$$
\begin{aligned}
& 0=a_{\infty}\left(\tilde{w} s a-\theta_{0}-\theta\right)-a, \\
& \dot{\theta}=\theta_{\infty}(a)-\theta, \\
& \dot{s}=\tilde{\tau}_{s}\left(s_{\infty}(a)-s\right) .
\end{aligned}
$$

where the dot represents now the derivative with respect to the slow time. The algebraic condition in (10) defines the critical manifold $S^{0,(a, \theta, s)}$ given 
in (9). Differentiating $S^{0,(a, \theta, s)}$ with respect to time, and projecting onto the $(a, s)$-space leads to the following system of differential equations:

$$
\begin{aligned}
-\left(\frac{-k_{a}}{a(1-a)}+\tilde{w} s\right) \dot{a} & =-\dot{\theta}+\tilde{w} a \dot{s}=-\left(\theta_{\infty}(a)-\theta\right)+\tilde{w} a \tilde{\tau}_{s}\left(s_{\infty}(a)-s_{\curlywedge}(1)\right. \\
\dot{s} & =\tilde{\tau}_{s}\left(s_{\infty}(a)-s\right),
\end{aligned}
$$

with $\theta$ constrained to be on $S^{0,(a, \theta, s)}$, that is

$$
\theta=k_{a} \ln \left(\frac{1}{a}-1\right)+\tilde{w} s a-\theta_{0}=\theta(a, s) .
$$

The singularities of equation (11) along the fold curve $F^{(a, \theta, s)}$ can be overcome through desingularization by applying a time rescaling of factor $\frac{k_{a}}{a(1-a)}-\tilde{w} s$, which gives the DRS:

$$
\begin{aligned}
& a^{\prime}=-\theta_{\infty}(a)+\theta(a, s)+\tilde{w} a \tilde{\tau}_{s}\left(s_{\infty}(a)-s\right), \\
& s^{\prime}=\left(\frac{k_{a}}{a(1-a)}-\tilde{w} s\right) \tilde{\tau}_{s}\left(s_{\infty}(a)-s\right) .
\end{aligned}
$$

Note that desingularization reverses the direction of flow on the repelling sheet of $S^{0,(a, \theta, s)}$ where $\frac{k_{a}}{a(1-a)}-\tilde{w} s<0$. Hence, the phase portrait of the reduced system is obtained by changing the direction of the flow in the phase portrait of the DRS where for $\frac{k_{a}}{a(1-a)}-\tilde{w} s<0$.

The structure of (12) does not allow symbolic calculation of the equilibria, but the computed bifurcation diagram with respect to $\tilde{w}$ shows the properties of its equilibria (Figure 8-(a)). Black and red branches of equilibria, one being stable the other unstable, exchange stability through a transcritical bifurcation at $\tilde{w}_{T} \approx 0.754645$. For $\tilde{w}<\tilde{w}_{T}$, the nodes of (12) are the true equilibria of (8) and the saddles are folded saddles. The solutions of (8) for $\tilde{w}<\tilde{w}_{T}$ are not affected by the folded saddles in this region because trajectories of (8) are attracted by the node equilibria. On the other hand, the nodes of (12) become folded nodes when $\tilde{w}>\tilde{w}_{T}$ because they are on $F^{(a, \theta, s)}$ (example phase portrait in Figure 8-(b)), while the saddles on the $s=s_{\infty}(a)$ curve turn into saddle-foci of the $3 \mathrm{D}$ system, with complex eigenvalues with positive real parts and one negative real eigenvalue. The presence of these two close equilibria (falls into the folded saddle-node type II [38]) is the footprint of the mechanism responsible for the small subthreshold oscillations: for big enough time-scale difference between the fast and slow components of the system, the orbits approach the folded node, around which there is as funnel structure $[11,26]$ that first attracts the orbits with a inward spiralling motion, and then repels them towards the $2 \mathrm{D}$ invariant manifold of the saddle of the full $3 \mathrm{D}$ system. Finally, the 2D unstable manifold of the saddle (associated with its the complex eigenvalues) provokes additional small oscillations just before the orbit leaves the vicinity of the critical manifold. 

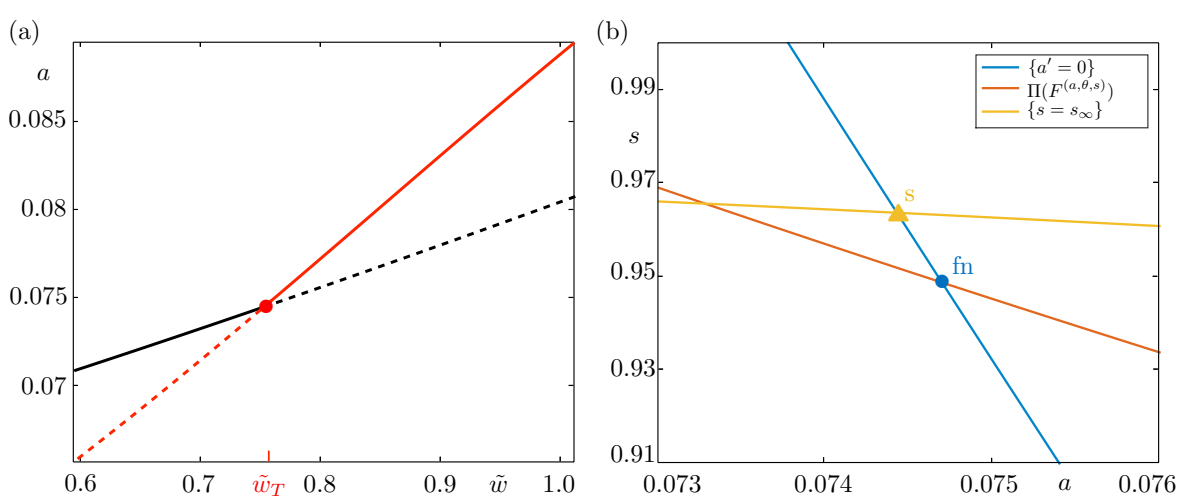

Fig. 8 (a) Bifurcation diagram of (12) with respect to $\tilde{w}$. Solid (resp. dashed) lines correspond to stable (resp. unstable) equilibria, and both branches cross at $\tilde{w}_{T}=0.754645$, where they exchange stability via a transcritical bifurcation (marked by a red dot); note that the branch plotted in red corresponds to the projection of the fold curve onto the $(a, s)$-plane. (b) Phase portrait of (12) for $\tilde{w}=0.7625$. The node equilibrium corresponds to a folded node $f n$ for the reduced system, with coordinates $\left(a_{\mathrm{fn}}, s_{\mathrm{fn}}\right) \approx(0.074696,0.94875)$; it lies at the intersection between the $a$-nullcline (blue) and the projection $\Pi\left(F^{(a, \theta, s)}\right)$ of the fold curve onto the $(a, s)$-plane (red). The saddle equilibrium s, with coordinates $\left(a_{\mathrm{S}}, s_{\mathrm{S}}\right) \approx(0.074426,0.96368)$, lies at the intersection between the $a$-nullcline (blue) and the $\left\{s=s_{\infty}\right\}$-component of the $s$-nullcline (yellow).

\subsection{MMOs and behaviour along isolas}

Figure 9-(a) shows the $\tilde{w}$-dependent bifurcation diagram of the $(a, \theta, s)$-model. The system undergoes a supercritical Hopf bifurcation at $\tilde{w}_{H o p f} \approx 0.755319$. The periodic solutions lie along the red branch starting at $\tilde{w}_{H o p f}$ grow in amplitude until $a_{\max }=1$, which are the relaxation type solutions studied in [39] (Figure 10-(b)).

The periodic solutions along the Hopf branch are unstable between two period doubling $(\mathrm{PD})$ bifurcations at $\tilde{w} \approx 0.758948$ and $\tilde{w} \approx 0.771919$. These unstable solutions are canard orbits that follow the unstable middle surface of $S^{0,(a, \theta, s)}$. The stable solutions between $\tilde{w} \approx[0.758948,0.771919]$ are MMOtype periodic orbits: $n$-number of SAOs near the folded node of $S^{0,(a, \theta, s)}$ followed by a LAO of relaxation type (Figure 7-(a)). All solutions with the same number of SAOs belong to the same isolated closed curve of periodic orbits, a so-called isola. Stable solutions lie on the top of each isola between two PD bifurcations on each. It is possible to pass from one isola to the next as $\tilde{w}$ changes within the approximate interval [0.758948,0.771919]. Transitions between neighbouring isolas corresponds to a \pm 1 change in the number of SAOs. The time course of some of these periodic orbits are given in Figure 10-(a).

The 9-SAO isola is displayed in Figure 9-(b) as an example. Observe that, the isolas present two high-slope segments between the bottom right corner (points 2 and 11) and the plateau on the top (see points 6 and 8 in the inset plot). We keep referring to them as the quasi-vertical segments of the solution branches. They connect the small-amplitude orbits to MMO-type orbits via 
canard explosions. Example periodic orbits on the left and right quasi-vertical segments are given in Figures 11 and 12, respectively. The orbit at the bottom left of the isola is in Figure 11-(1). This subthreshold orbit has the shortest segment along the repelling surface and it has 9 SAOs plus one last oscillation slightly larger than the others. When moving $\tilde{w}$ on the left quasi-vertical segment, the associated cycle extends the part of its last oscillation along the repelling middle sheet of $S^{0,(a, \theta, s)}$ (the orbits on panels (1)-(3) are similar to the canard-without-head cycles in planar systems). The maximal canard trajectory (the third orbit) is the solution that stretches out from the lower side of the fold curve all the way to the upper side. As $\tilde{w}$ changes, the solution follows the repelling sheet of the critical manifold, before jumping up to the upper attracting sheet, then jumping down to the lower attracting branch and finally funnels into the folded-node region (the forth and fifth orbits are similar to the canard-with-head cycles in planar systems). The formation of the relaxation segment of the periodic solution (the LAO) is completed when $\tilde{w}$ passes the first PD bifurcation point (Figure 11-(6)), where the solutions become stable.

As $\tilde{w}$ continues to move along the isola, periodic orbits lose their stability at the second PD point, then they shrink in amplitude while the parameter goes down along the right quasi-vertical segment. Here we observe a second canard explosion, which happens in the opposite direction as the usual one (in a sense, a canard implosion), connecting the MMOs to the small-amplitude periodic orbits (Figure 12). We see that the only difference between the solutions on the left and right quasi-vertical segments is the amplitude of the 9th SAO, which is slightly larger than the ones along the left quasi-vertical segment.

We also observe that the silent phase duration increases with the number of SAOs, i.e. when decreasing the value of $\tilde{w}$ (Figure 10-(a)). The silent phase can take up to 12 times longer than the active phase for the MMOs, whereas the maximum ratio for the relaxation type orbits (Figure 10-(b)) is around 5 . This provides a new hypothesis to explain the large silent to active phase duration ratio observed experimentally.

The bifurcation structure of this model, in particular the family of isolas of MMOs, their shape and organization in parameter space, is very much exemplary of excitable systems with one fast and two slow variables near a folded node. Indeed, such bifurcation structure has been found in various single neuron models (e.g. in the reduced Hodgkin-Huxley model) and also in models of chemical reactions such as the Koper model; see [11] for details and further examples.

\section{Canard-mediated MMBOs oscillations in the $(a, d, \theta, s)$-model}

In Section 2 we tracked the $s$-dependent family of canard cycles in the $(a, d, \theta)$ burster. In Section 3, we detailed the MMO behaviour formed by an alternation between canard-mediated subthreshold oscillations and one LAO in the $(a, \theta, s)$-model. In this section, we combine the bursts with MMOs in the 4D system (4), and focus on the canard trajectories of the resulting MMBOs. We 
(a)

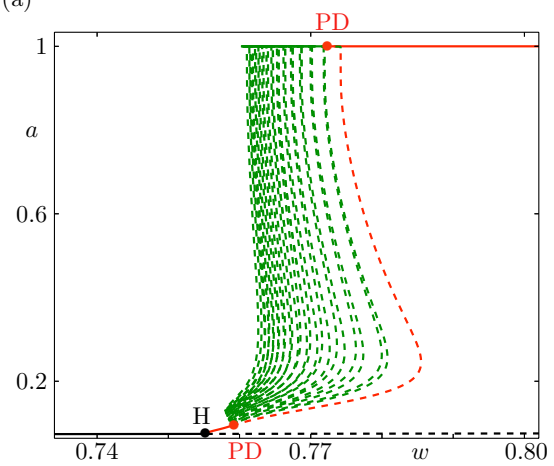

(b)

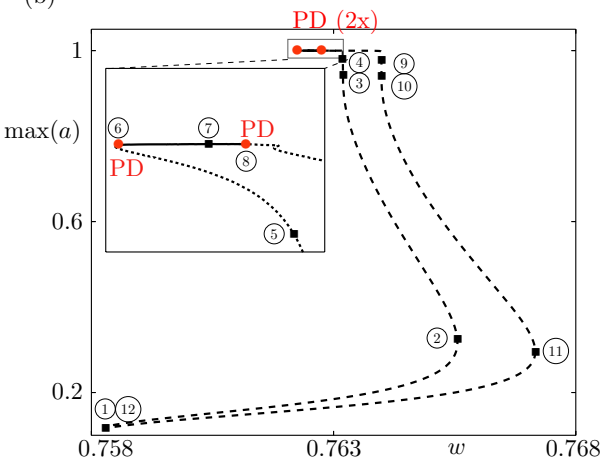

Fig. 9 (a) Bifurcation diagram of the $(a, \theta, s)$-model with isolas of MMO solutions. (b) Zoom of panel (a) near the 9-SAO-isola. The inset panel offers a further zoom into the upper region of the isola. Bold lines correspond to stable solutions, dashed lines represent unstable ones. In all panels, red (resp. black) dots indicate period-doubling (resp. Hopf) bifurcations. In panel (b) and its inset, marked solutions are shown in Figures 11 and 12.

(a)

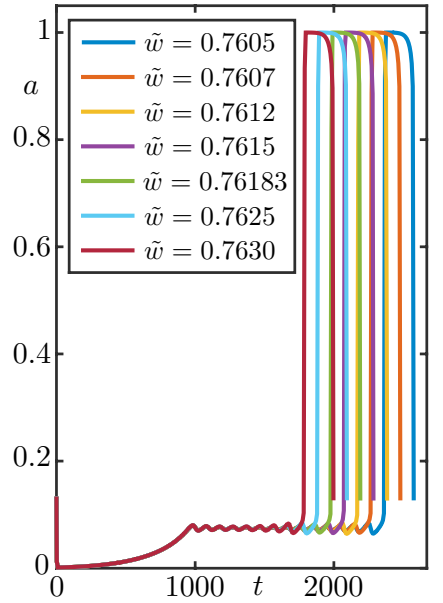

(b)

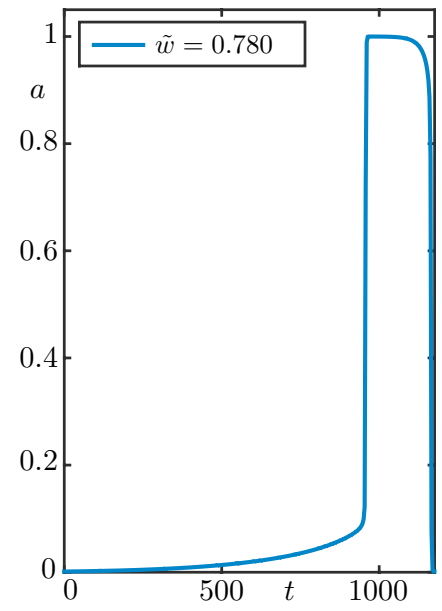

Fig. 10 (a) Example MMO-type solutions of the $(a, \theta, s)$-model for $\tilde{w} \in$ $[0.758948,0.771919]$. (b) A relaxation-type solution of the $(a, \theta, s)$-model for $\tilde{w}=0.78$.

consider the network connectivity parameter, $w$, as the main bifurcation parameter of (4), while other parameters are chosen as in [41]. This is a quite reasonable assumption since the model represents a developmental process where the network connectivity changes.

We start by investigating the slow flow near the critical manifold (4). The critical manifold is a surface in $\mathbb{R}^{4}$, defined by the algebraic equation:

$$
\begin{aligned}
S^{0} & :=\left\{a_{\infty}\left(w d s a-\theta-\theta_{0}\right)-a=0, d_{\infty}(a)-d=0\right\} \\
& :=\left\{k_{a} \ln \left(\frac{1-a}{a}\right)+w d_{\infty}(a) s a-\theta-\theta_{0}=0\right\} .
\end{aligned}
$$


(1)
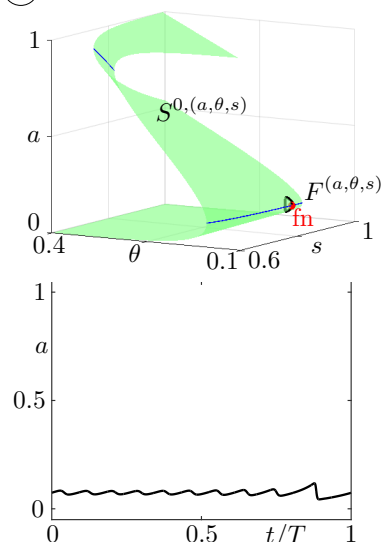

(4)
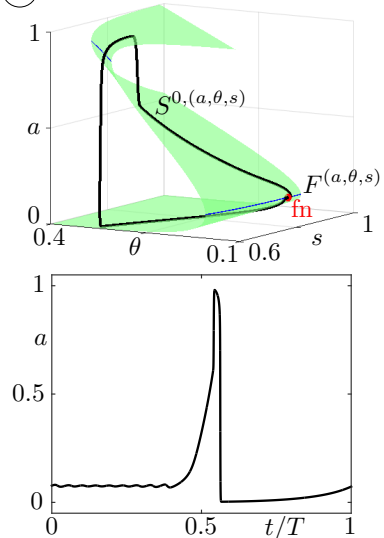

(2)
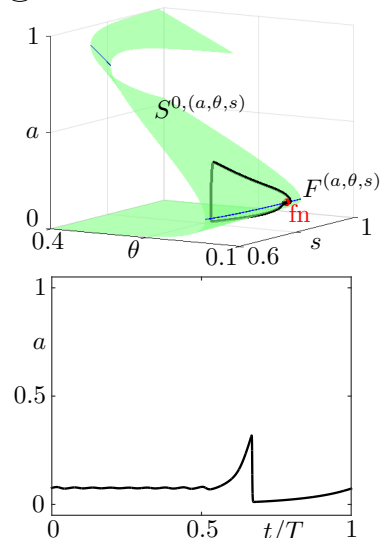

(5)
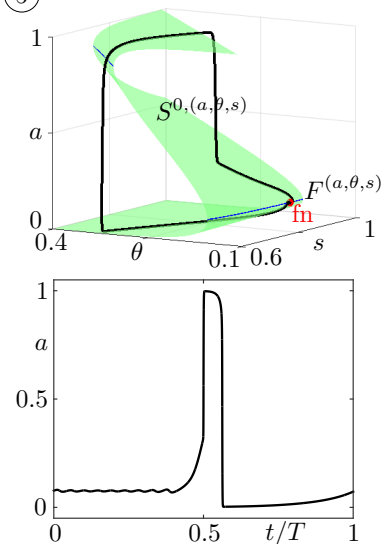

(3)
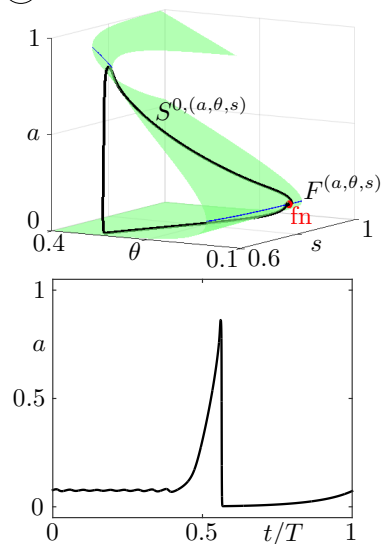

(6)
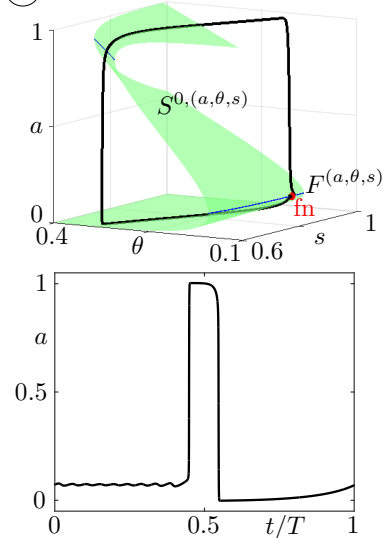

Fig. 11 Upward behaviour of the orbits when $w$ moves along the left quasi-vertical segment of the isola on panel (b) of Figure 9. A canard explosion connects in parameter space a SAO periodic orbit (upper left) to a MMO periodic orbit (bottom right). The green surface is the critical manifold $S^{0,(a, \theta, s)}$, the blue lines on $S^{0,(a, \theta, s)}$ correspond to the single fold curve $S^{0,(a, \theta, s)}$ (the critical manifold is in fact a cusp surface). Each panel below the $(a, \theta, s)$-space projection shows the time course for $a$ of the periodic orbit shown above.

Since two slow variables, $\theta$ and $s$, appear in the expression of $S^{0}$, the slow flow is $2 \mathrm{D}$ on the critical surface. The critical manifold $S^{0}$ is folded along the folded curve $F$ :

$$
F=\left\{\frac{-k_{a}}{a(1-a)}+w s\left(a d_{\infty}^{\prime}(a)+d_{\infty}(a)\right)=0\right\} .
$$


(7)
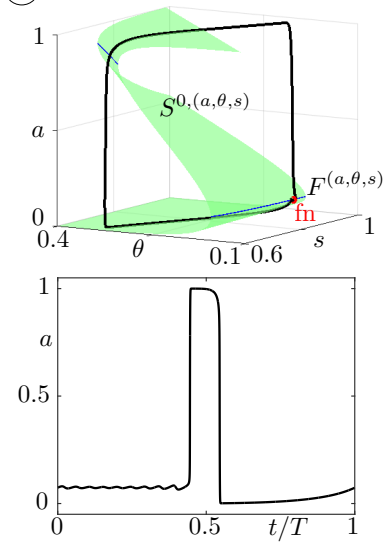

(10)
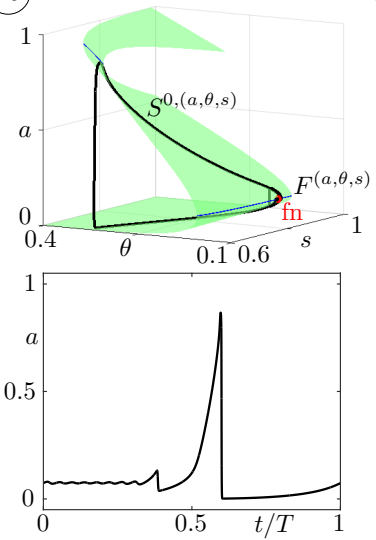

(8)
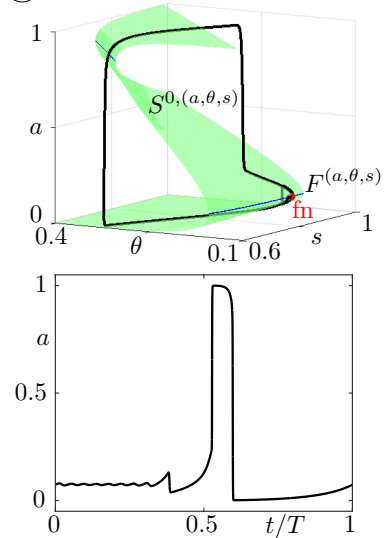

(11)
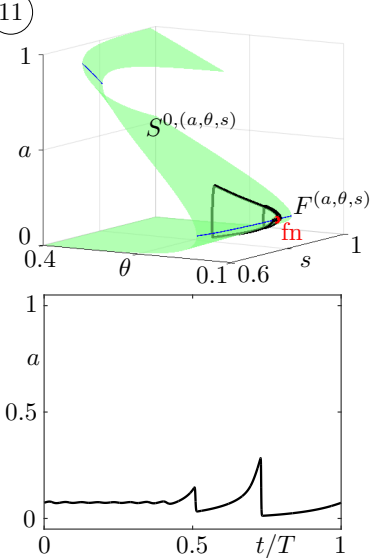

(9)
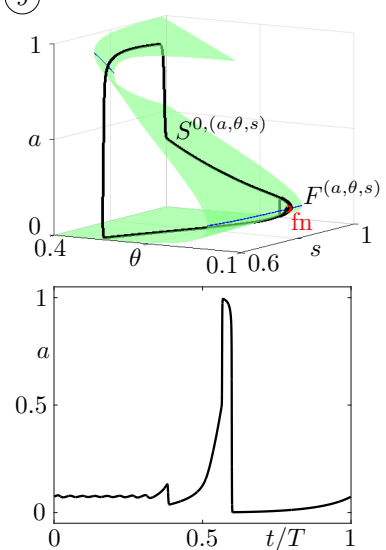

(12)
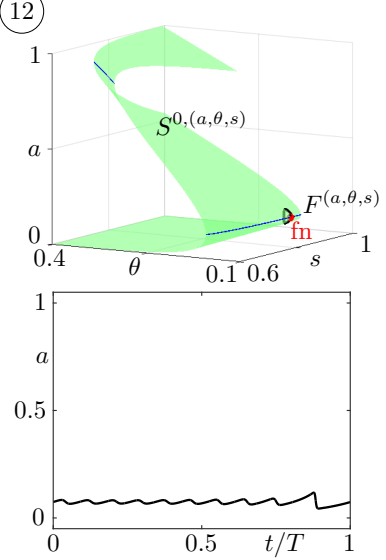

Fig. 12 Downward behaviour of the orbits when $w$ moves along the right quasi-vertical segment of the isola on panel (b) of Figure 9. A canard implosion connects in parameter space the MMO periodic orbit (upper left) to SAO periodic orbit (bottom right). The green surface is the critical manifold $S^{0,(a, \theta, s)}$, the blue lines on $S^{0,(a, \theta, s)}$ correspond to the single fold curve $S^{0,(a, \theta, s)}$ (the critical manifold is in fact a cusp surface). Each panel below the $(a, \theta, s)$-space projection shows the time course for $a$ of the periodic orbit shown above.

Keeping $\theta$ on $S^{0}$ by setting $\theta=-\theta_{0}+k_{a} \ln \left(\frac{1-a}{a}\right)+w s a d_{\infty}(a)$, the DRS is given by

$$
\begin{aligned}
& a^{\prime}=-\left(\theta_{\infty}(a)-\left(-\theta_{0}+k_{a} \ln \left(\frac{1-a}{a}\right)+w s a d_{\infty}(a)\right)\right)+\tilde{\tau}_{s} w a d_{\infty}(a)\left(s_{\infty}(a)-s\right) \\
& s^{\prime}=-\left(\frac{-k_{a}}{a(1-a)}+w s\left(a d_{\infty}^{\prime}(a)+d_{\infty}(a)\right)\right) \tilde{\tau}_{s}\left(s_{\infty}(a)-s\right) .
\end{aligned}
$$

Note that since the dynamics of $d$ can be expressed as a function of $a$ in (13), i.e. $d=d_{\infty}(a)$, the most general way to derive DRS given in (3) is not necessary. 

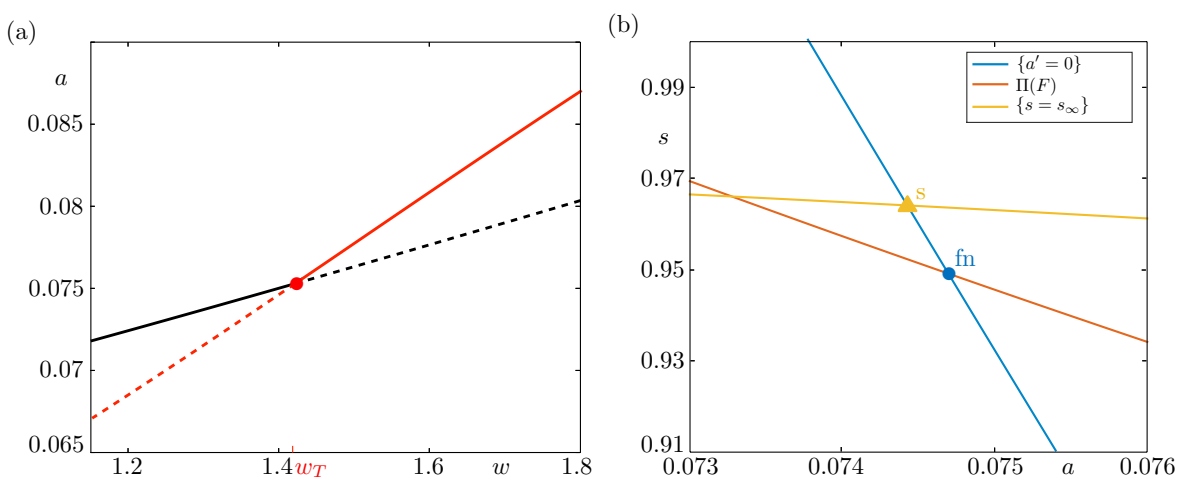

Fig. 13 (a) Bifurcation diagram of (14) with respect to $w$. Solid (resp. dashed) lines correspond to stable (resp. unstable) equilibria, and both branches cross at $w_{T}=1.4218$, where they exchange stability via a transcritical bifurcation; note that the branch plotted in red corresponds to the projection of the fold curve onto the $(a, s)$-plane. (b) Nullclines of (14) for $w=1.43$ : in blue, the $a$-nullcline; in red and yellow, both components of the $s$-nullcline. Also shown are the two equilibria of the system: the saddle equilibrium $s$ with coordinates $\left(a_{s}, s_{s}\right) \approx(0.0753962,0.962052)$ lies at the intersection between the $a$-nullcline (blue) and the $\left\{s=s_{\infty}\right\}$-component of the $s$-nullcline (yellow); the folded-node $\mathrm{fn}$ with coordinates $\left(a_{\mathrm{fn}}, s_{\mathrm{fn}}\right) \approx(0.0755447,0.954177)$, lies at the intersection between the $a$-nullcline (blue) and the projection $\Pi(F)$ of the fold curve onto the $(a, s)$-plane (red).

The bifurcation diagram of (14) with respect to $w$ is presented in Figure 13(a). Similar to the one of (12), two sets of equilibria of saddle- and node-types (red and black curves) undergo a transcritical bifurcation at $w_{T} \approx 1.4218$ (a folded saddle-node type II event). For $w>w_{T}$, the nodes of the DRS, $\left(a_{f}, s_{f}\right)$, lie on the folded curve $F$ and correspond to folded-node singularities of (4) (see an example of phase portrait on Figure 13-(b)). On the other hand, the saddle-type equilibria, $\left(a_{s}, s_{s}\right)$ satisfy $s=s_{\infty}(a)$, thus they are also equilibria of the full system (4). In this region of parameter space (i.e. $w>w_{T}$ ) and for small enough values of the timescale separation parameter $\varepsilon$, then the MMO trajectories of (4) can be trapped by the funnel surrounding the folded node. They first make SAOs inside the funnel towards the folded node, pass near it and then are likely to continue displaying SAOs close to the repelling side of $S^{0}$ along the 1D attracting manifold of the saddle equilibrium of the full system, before being repelled by spiralling away along the 2D unstable manifold of the saddle. This $2 \mathrm{D}$ unstable manifold associated with the complex eigenvalues of the saddle hence provokes additional small oscillations just before the orbit leaves the vicinity of the critical manifold and enters into the burst phase. Note that resulting periodic orbits with SAOs and bursting oscillations, that is MMBOs, are possible in system with at least 2 fast and 2 slow variables.

\subsection{MMBOs and behaviour along isolas}

Figure 14 shows the bifurcation diagram of the $(a, d, \theta, s)$-model with respect to parameter $w$. At $w_{H} \approx 1.42122$ the system undergoes a supercritical Hopf 


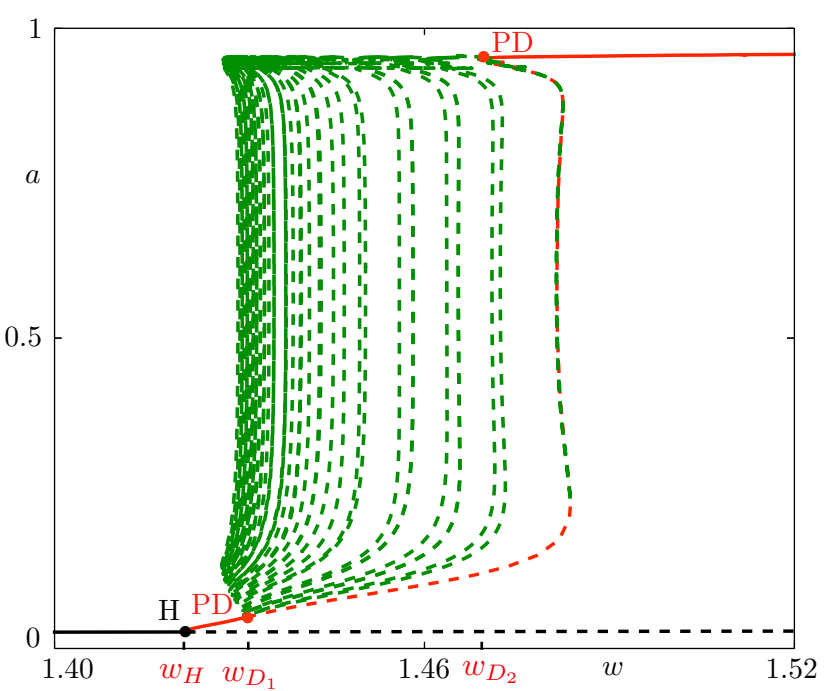

Fig. 14 Bifurcation diagram of the $(a, d, \theta, s)$-model with isolas of the MMBO solutions solid lines correspond to stable solutions, dashed lines to unstable ones. Red dots indicate PD bifurcations and the black dot corresponds to the Hopf bifurcation. The branch of equilibria (black) undergoes a Hopf bifurcation at $w_{H} \approx 1.42122$, which gives rise to a branch of periodic solutions (red) which is unstable between the PD bifurcations at $w_{D_{1}} \approx 1.42748$ and $w_{D_{2}} \approx 1.47011$. Isolas of MMBO-type periodic oscillations are shown in green.

(a)

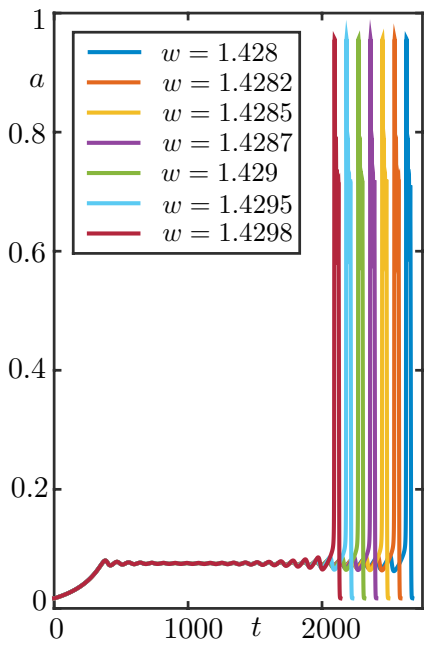

(b)

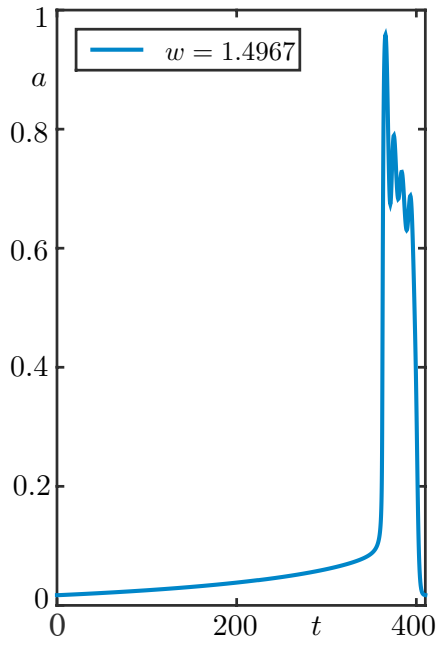

Fig. 15 (a) Example of MMBO-type solutions of the $(a, d, \theta, s)$-model for $w$ in the approximate interval $[1.428,1.47]$. Each solution corresponds to particular isola from Figure 14. (b) Bursting behaviour (without SAOs) of the $(a, d, \theta, s)$-model for $w=1.4967$. 
bifurcation, where a branch of periodic solutions of bursting type arises (Figure 15-(b)). Periodic solutions along this branch are unstable between two PD bifurcations at $w \approx 1.43103$ and $w \approx 1.47011$. The stable solutions of the system between these two PD bifurcations are the MMBO-type orbits that reside on isolas in $w \approx[1.42748,1.47011]$ (see example solutions in Figure 15-(a)). The transcritical bifurcation of $(14)$ at $w_{T} \approx 1.4218$ can be considered as a lower bound for MMBO dynamics as $\varepsilon \rightarrow 0$. The isolas are more densely positioned for $w$-values closer to $w_{H}$. The last isola overlaps with the branch of the bursting-type solutions (blue curve superimposed on the red branch arising at the Hopf point). Each isola corresponds to a particular sector of rotation of canard solutions near the folded node yielding SAOs, and each periodic orbit with $n$-SAOs lies on a different isola. A variation of $w \in[1.42748,1.47011]$ can move an orbit from one sector to another and, consequently, change the number of SAOs of the solution (Figure 15-(a)). We show a representative selection of the family of isolas but there may be more of them. Given that each isola can be associated with a given number of SAOs, one can obtain a prediction of the number of isolas by computing the maximal rotation number given by the ratio of the eigenvalues of the folded node (as a node equilibrium of the DRS); see [11] for details. This is only a prediction valid for small-enough $\varepsilon>0$. This number, and hence the number of isolas, will increase up to infinity as the folded node approaches a folded saddle-node transition. In the present work, we do not to expand on these questions.

Figure 16 shows the isola formed by the 18-SAO-orbits. Along the isola, canard trajectories organise the transition from the "pure" subthreshold SAOsolutions to spiking, and then to MMBO-type solutions (similar to the spikeadding canard explosion in the (a,d, $\theta)$-model). The orbit in Figure 17-(1) has 18 SAOs plus one last SAO containing a canard segment with larger amplitude. As $w$ varies following the left branch of the isola, that canard segment expands along the middle sheet of $S^{0}$. It grows until the upper fold of $S^{0}$ and becomes a maximal canard segment (which approximates the spiking threshold) of the left branch of the isola (Figure 17-(3)). When the orbit reaches the upper fold of $S^{0}$, the stable and unstable manifold of the associated slow manifold expand and contract it in the $a$-direction. Finally the orbit crosses the middle branch (the spiking threshold [40]) and the first spike appears Figure 17-(4)). As $w$ decreases, the canard segment shrinks while the formation of the spike continues. The solution with a fully formed spike has 18 SAOs and 1 LAO (Figure 17-(6)) and it lies on the left corner of the quasi-horizontal branch of the isola that has a sharp fold at $a_{\max } \approx 0.94$ (Figure 16-(b)).

As $w$ changes, the orbits move leftwards along the lower branch emanating form the sharp turning point at $a_{\max } \approx 0.94$ and the second canard segment, which is located after the spike, expands; see orbits (6) to (8) on Figure 16-(b). The solution with one spike and the longest canard segment corresponds to orbit (8) and just after, a new spike appears. As we move along the upper fold, the canard segment between two spikes shrinks. The solution becomes a periodic orbit with $18 \mathrm{SAOs}$ and 2 spikes when it reaches the left fold point of the second quasi-horizontal segment of the branch around $a_{\max } \approx 0.948$ 
((9) on Figure 16-(b)). The third and fourth spikes are formed through the same principles. The stable solutions with four spikes lie between the orbit (15) and the PD point on the top horizontal segment of the isola. After the PD bifurcation, orbits become unstable and lose spikes as $w$ varies towards the bottom of the isola along its right branch - similar to the canard implosion described in Section 3. Note that experimentally the number of spikes per burst increases as the embryos develop. Early in development, it seems that spikes are added in a similar way as described here, i.e. the new spike first occurs just at the end of a burst, then "moves to the left". Some evidence for this is shown in [40, Figure 5].

The period of a canard cycle is closely related to the length of its repelling segment $[10,21]$. Along a canard explosion in a planar VDP-type system, the period function is increasing between the Hopf bifurcation and the maximal canard cycle, and it is decreasing from the maximal canard to the relaxation regime. Thus the maximal canard cycle, being the solution with the longest repelling segment, has the longest period [10]. This property holds in MMBOs as well. Figure 16-(c) shows the period plotted against $w$ along one of the isolas just described; it clearly has a complex leaf-structure with multiple local maxima. The behaviour of the period along each leaf resembles a "local" canard explosion: orbits on each local maximum (orbits $3,8,11,14$ ) correspond to maximal canard solutions of each leaf, and the orbits $(1,6,9,12,15)$ on local minima have the shortest canard segments. Among these solutions, orbit (1) corresponds to the global minimum of the isola, therefore it the "fastest" solution with 18 SAOs.

As in the MMO-type solutions of the $(a, s, \theta)$-model, the MMBO-type orbits with more SAOs have longer silent phases without any significant change in the active phase duration. The ratio of silent phase over active phase durations changes approximately fourfold (between 13 and 50) for the MMBOs whereas the maximum ratio for the bursting solutions $\left(w>w_{D_{2}}\right.$, see Figure 14 ) is around 9.

\section{Conclusion}

We have revisited a group of related rate models introduced and analysed in $[41,40,39]$ from the viewpoint of multiple-timescale dynamics and complex oscillations. The complete system contains 4 variables (2 fast and 2 slow), where the fast subsystem corresponds to a recurrent excitatory network with fast activity-dependent synaptic depression, which can produce oscillations [41]. In this work, we have considered three different combinations of the 4 variables and analysed them using notions from GSPT. In particular, we have focused on the excitable structure of each model and shown how it leads to canard solutions. Adding either the dynamics of the cell firing threshold $((a, d, \theta)$-model $)$ or long-term synaptic depression $((a, d, s)$-model $)$, bursting oscillations are generated [41]; we have described them through their canardmediated spike-adding structure [11]. 

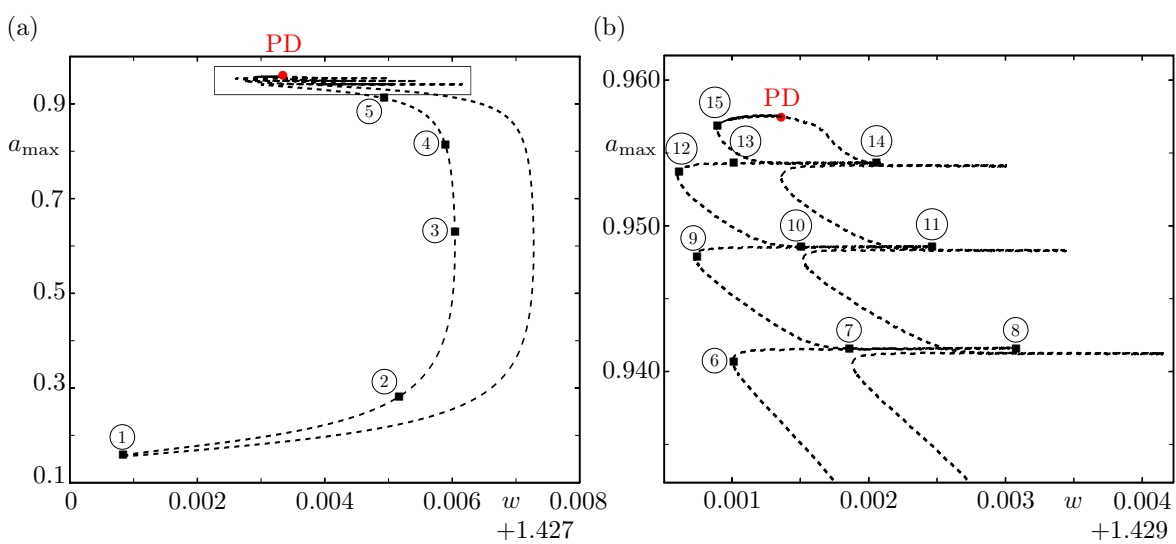

(c)

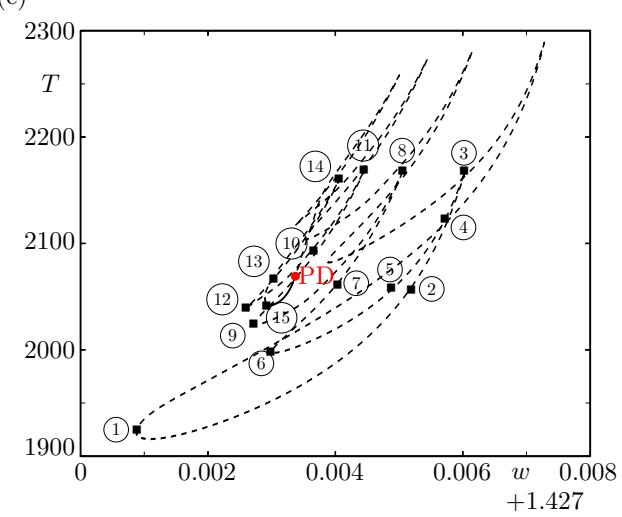

Fig. 16 (a) The isola corresponding to MMBO orbits with 18 SAOs. (b) Zoom into the upper branch of the isola highlighted as an inset in panel (a). (c) Period along the isola shown in panel (a). The profile of the solutions marked by a black square are shown in Figures 17-18. In all panels, solid curves correspond to stable solutions while dashed curves correspond to unstable solutions.

We have also investigated the combined effect of the threshold for cell firing and the long-term synaptic depression on the network activity by studying the slow flow associated to the $(a, \theta, s)$-model, which turns out to display canardinduced MMOs due to the presence of a folded-node singularity. Moreover, we have been able to understand the structure of families of MMOs organised in parameter space along isolas, as one typically expects from such slow-fast systems.

Finally, we have gathered all four variables in the complete $(a, d, \theta, s)$ model, which displays complex oscillations that can be described as a mix between MMOs and bursting. These fall into the category of MMBOs introduced in [12]. We computed for the first time the remarkable bifurcation structure of MMBOs, also organised in families of isolas that retain the salient features of both the isolas of MMOs and of bursting oscillations from the associated aforementioned 3D systems. In each case, isolas can be understood as a way 

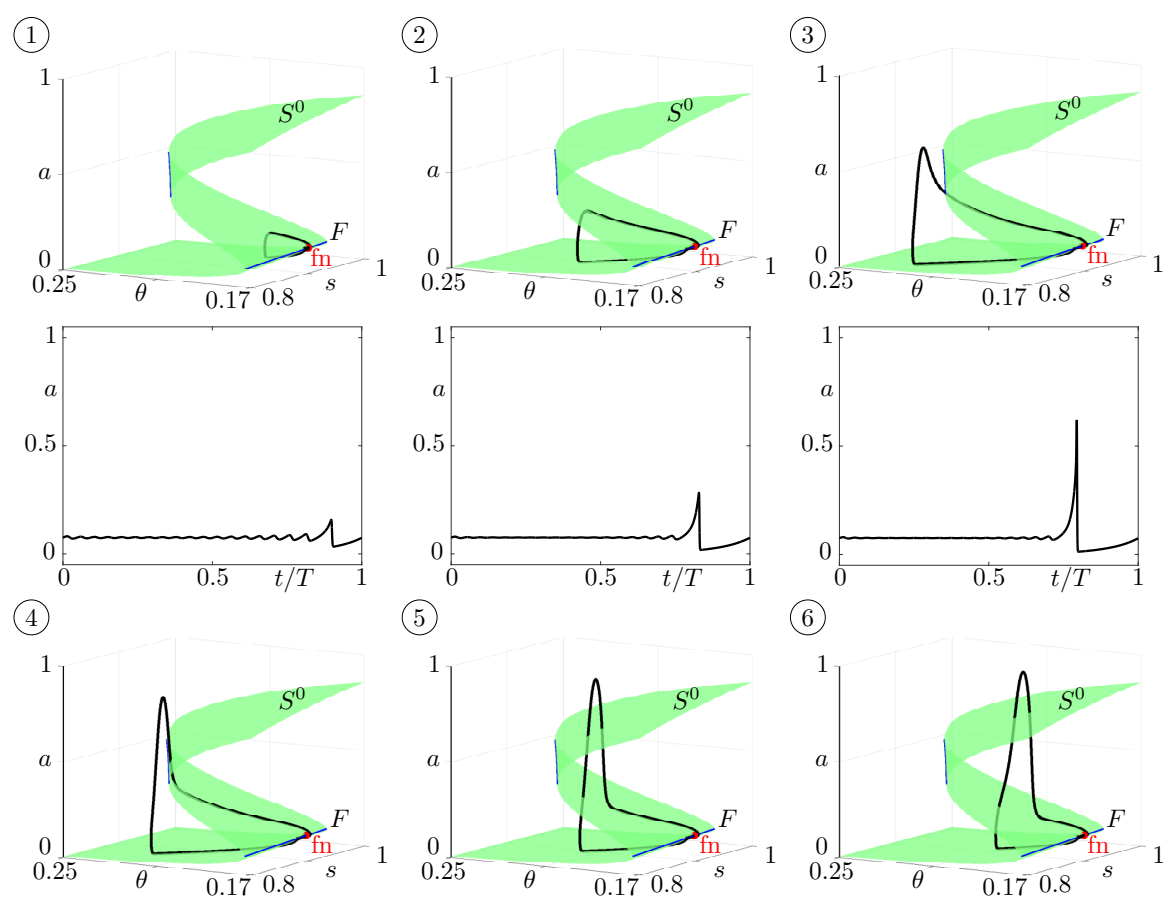

(5)

(6)
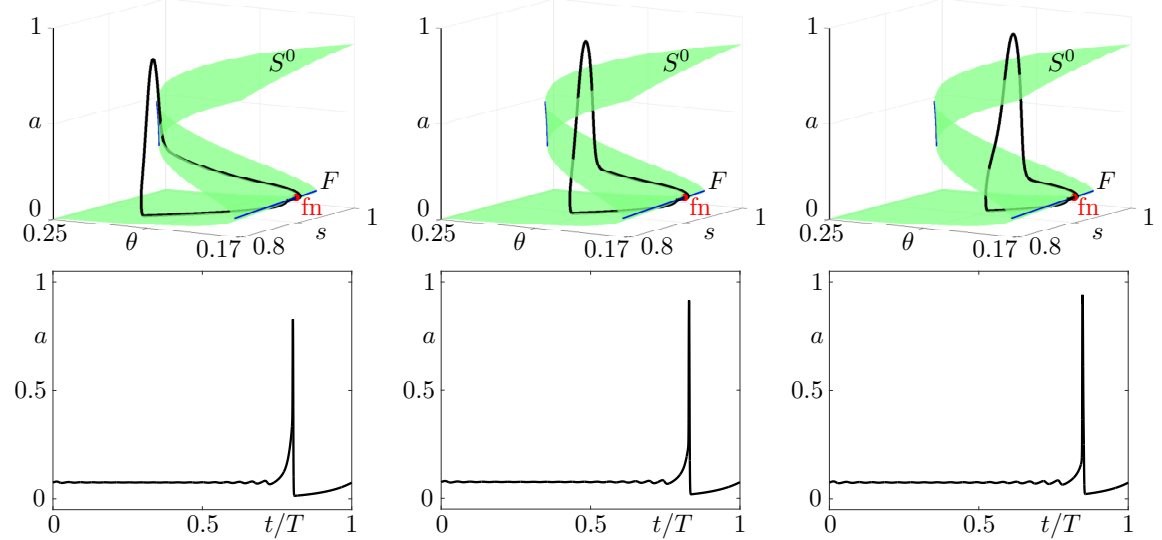

Fig. 17 Birth of the first canard-mediated spike along the isola corresponding to MMBO solutions with 18 SAOs in Figure 16 as $w$ varies. Each up-down pair shows the solution projected onto the $(\theta, s, a)$-space (upper panel) and the $a$-time course of the solution (lower panel). The green surface is the critical manifold $S^{0}$ and the blue curve is the lower fold $F$, the red dot marks the folded node fn.

for the system to single-out specific oscillatory patterns. Moreover, we qualitatively analyse the profile of MMBO solutions along one such isola, which reveals canard-mediated transitions that affect the number of subthreshold oscillations as well as the number of spikes per burst for MMBOs on this isola. Our analysis suggests that multiple slow processes are worth considering in modelling studies investigating noisy subthreshold fluctuations, which could unveal further physiological features.

We know from the experimental recordings that stochasticity plays a role in triggering episodes of activity, while termination of an episode is consistent with deterministic dynamics. But it is also very likely that there are mechanisms of depression that include both synaptic depression $(s)[9,16]$ and adaptation of neuron excitability $(\theta)[1,46]$. The (deterministic) MMBO framework 
(7)
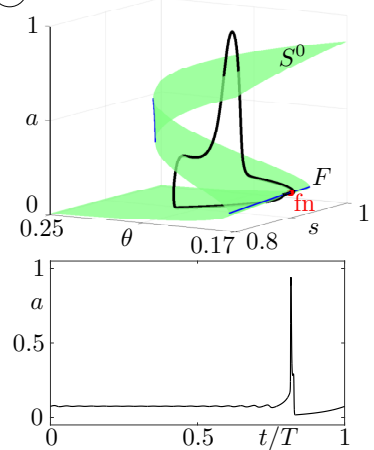

(10)
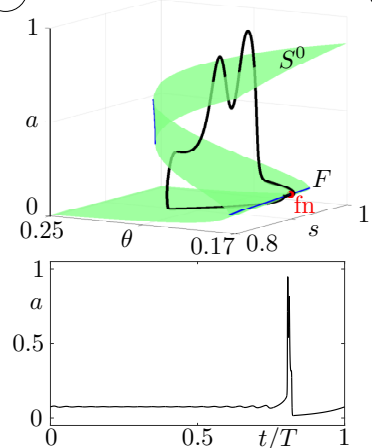

(13)
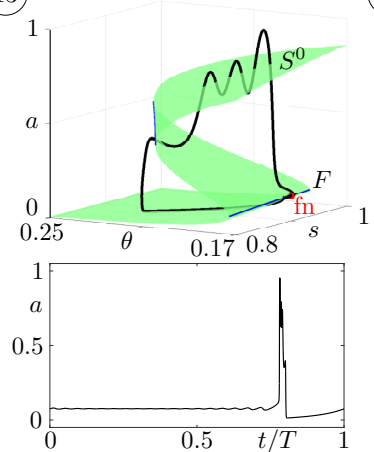

(8)
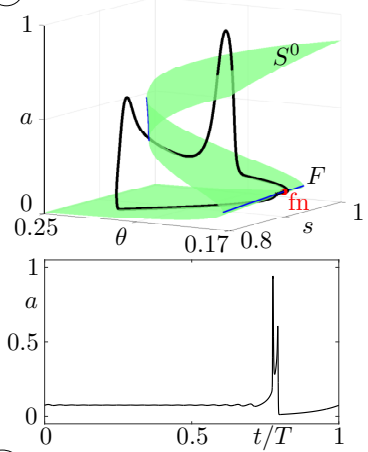

(11)

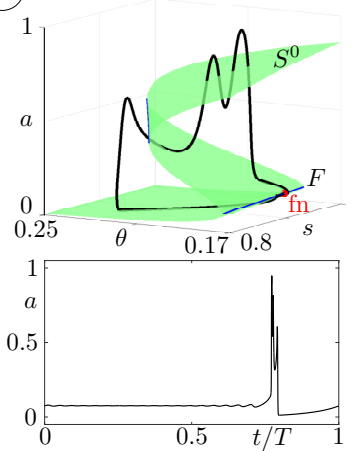

(14)

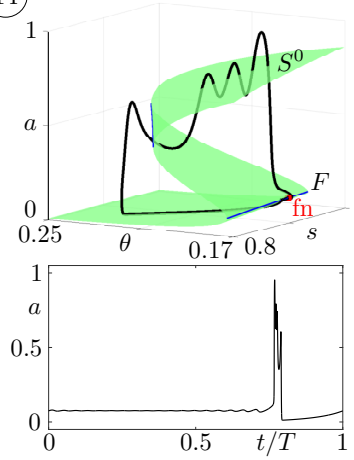

(9)
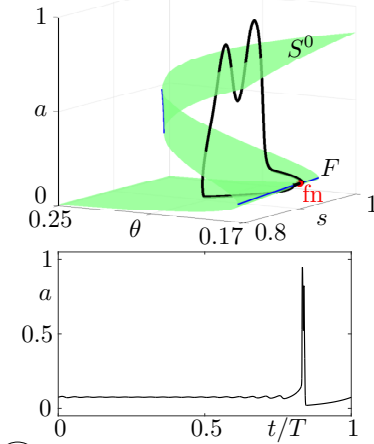

(12)
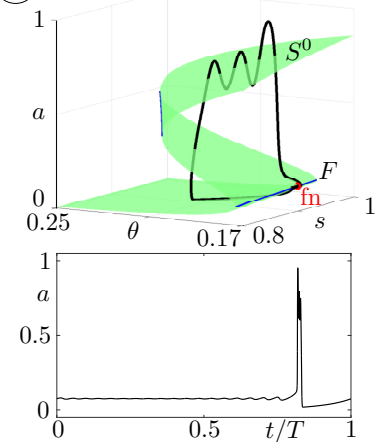

(15)
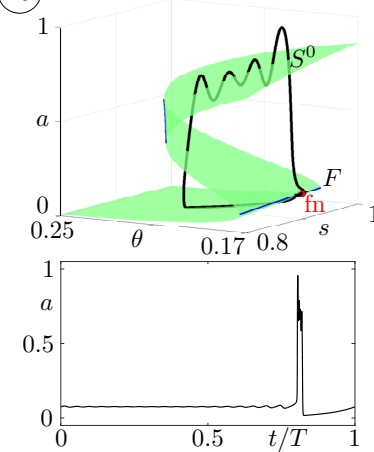

Fig. 18 The second, third and fourth canard-mediated spikes along the isola corresponding to MMBO solutions with 18 SAOs. Each up-down pair shows the solution on the $(\theta, s, a)$ space (upper panel) and the time corse of the solution (lower panel). The green surface is the critical manifold $S^{0}$ and the blue curve is the lower fold $F$, the red dot marks the folded node fn. 
we developed here showed that the consequence of having the two slow variables, at least in some part of parameter space, is that there are SAOs during the silent phase, which turn out to be relevant in setting the length of the silent phase and shaping the subthreshold transients. In other words, in developing networks noise affects the silent phases' terminations, while SAOs prolong silent phases by tailoring subthreshold dynamics. This leads to the question of who wins, noise or SAOs? Perhaps SAOs temper the effects of noise (Berglund et al. have shown that canard-mediated SAOs can survive in a noisy environment [4]), or perhaps noise masks the canard structure that gives rise to SAOs. The available experimental data are too scarce to formulate hard conclusions, however future work should address possible interactions between noise and canards that would lead to interesting mathematics and modelling aspects.

A key parameter in our bifurcation analysis is the level of connectivity within the network, directly related to development of the chick spinal cord. It has been shown that changes in the connectivity affect the duration of both bursting and silent states (see for instance Fig. 13 in [41]). We show that the presence of SAOs in the solution increases considerably the ratio between the silent and active phase durations. Our dissection of the bifurcation structure of MMBOs reveals as well the emergence of a complex substructure for intermediate connectivity levels: the control on the families of isolas allows to match in a precise way the connectivity parameter with the number of spikes in the burst and the number of SAOs. Although the range of the network connectivity yielding MMBOs is small here, it can be increased for different parameter sets (for instance by taking $\tau_{s} \approx \tau_{\theta}$ ). A more extensive study of the parameter space will be an interesting topic for follow-up work.

Overall, the main interest of the slow-fast approach developed in the present work lies in its ability to map the boundaries between different activity regimes (pure spiking, spiking with subthreshold oscillations, bursting, bursting with subthreshold oscillations, etc.) and different oscillatory patterns within each regime. These are understood through a theoretical prism which allows to identify the organising centres of these boundaries (folded singularities, maximal canards) as well as key (combinations of) parameters to control them (e.g. the number of rotation sectors near a folded node).

Another important novelty of the work is that it provides one of the first studies of canard-induced complex oscillations in rate models for neural network dynamics. Several follow-up directions can be envisaged. First, coupling general excitatory networks to inhibitory ones and analysing similar slow-fast complex oscillatory dynamics in the resulting excitatory/inhibitory systems. In particular, it will be interesting to investigate how multiple-timescale interactions between the different components of such coupled systems may provoke a sudden break-up of the excitation/inhibition balance, known to trigger transitions to certain pathological brain states (such as epileptic seizures). The same paradigm can be also useful to understand phenomena like the emergence of bursting activity in connected neuronal sub-populations, see [6], or the change in network dynamics related to changes in the effectiveness of synaptic depression, see [2]. In particular, how the dynamics of a network can turn from 
up- and down-states into asynchronous states while increasing the strength of short-term depression is worth to investigate. Another future direction will consist in finding a similar slow-fast taxonomy of canard-induced complex oscillations in networks of spiking neurons and compare them with the firing rate activity that we have observed here.

\section{References}

1. Bazzara, D., Kita, H., J., W.C.: Slow spike frequency adaptation in neurons of the rat subthalamic nucleus. J. Neurophysiol. 102(6), 3689-3697 (2009)

2. Benita, J., Guillamon, A., Deco, G., Sanchez-Vives, M.: Synaptic depression and slow oscillatory activity in a biophysical network model of the cerebral cortex. Frontiers in Computational Neuroscience 6, 64 (2012). DOI 10.3389/fncom.2012.00064. URL https://www.frontiersin.org/article/10.3389/fncom.2012.00064

3. Benoît, E., Callot, J.L., Diener, F., Diener, M.: Chasse au canard. Collect. Math. 32(12), 37-119 (1981)

4. Berglund, N., Gentz, B., Kuehn, C.: Hunting French ducks in a noisy environment. J. Differ. Equ. 252, 4786-4841 (2012)

5. Bertram, R., Rhoads, J., Cimbora, W.P.: A phantom bursting mechanism for episodic bursting. Bulletin of Mathematical Biology 70(7), 1979-1993 (2008)

6. Bisio, M., Bosca, A., Pasquale, V., Berdondini, L., Chiappalone, M.: Emergence of bursting activity in connected neuronal sub-populations. PLOS ONE 9(9), 1-14 (2014). DOI 10.1371/journal.pone.0107400. URL https://doi.org/10.1371/journal.pone.0107400

7. Brøns, M., Krupa, M., Wechselberger, M.: Mixed-mode oscillations due to the generalized canard mechanism. Fields Inst. Commun. 49, 39-63 (2006)

8. Burke, J., Desroches, M., Barry, A.M., Kaper, T.J., Kramer, M.A.: A showcase of torus canards in neuronal bursters. J. Math. Neurosci. 2(3) (2012)

9. Chub, N., O'Donovan, M.J.: Journal of neurophysiology logo journal home issues collectionsinformationabout submitsubscribe quick search in journals search this journal article post-episode depression of GABAergic transmission in spinal neurons of the chick embryo. J. Neurophysiol. 85(5), 2166-2176 (2001)

10. De Maesschalck, P., Desroches, M.: Numerical continuation techniques for planar slowfast systems. SIAM J. Appl. Dyn. Syst. 12(3), 1159-1180 (2013)

11. Desroches, M., Guckenheimer, J., Krauskopf, B., Kuehn, C., Osinga, H.M., Wechselberger, M.: Mixed-mode oscillations with multiple time scales. SIAM Rev. 54(2), 211288 (2012)

12. Desroches, M., Kaper, T.J., Krupa, M.: Mixed-mode bursting oscillations: Dynamics created by a slow passage through spike-adding canard explosion in a square-wave burster. Chaos 23, 046106 (2013)

13. Desroches, M., Krupa, M., Rodrigues, S.: Inflection, canards and excitability threshold in neuronal models. J. Math. Biol. 67, 989-1017 (2013)

14. Desroches, M., Krupa, M., Rodrigues, S.: Spike-adding in parabolic bursters: The role of foded-saddle canards. Physica D 331, 58-70 (2016)

15. Ermentrout, B., Terman, D.: Mathematical Foundations of Neuroscience. Springer (2010)

16. Fedirchuk, B., Wenner, P., Whelan, P., Ho, S., Tabak, J., O’Donovan, M.J.: Spontaneous network activity transiently depresses synaptic transmission in the embryonic chick spinal cord. J. Neurosci. 19, 2102-2112 (1999)

17. Fenichel, N.: Asymptotic stability with rate conditions II. Indiana Univ. Math. J. 26, 81-93 (1977)

18. Guckenheimer, J., Kuehn, C.: Computing slow manifolds of saddle type. SIAM J. Appl. Dyn. Syst. 8(3), 854-879 (2009)

19. Izhikevich, E.M.: Neuronal excitability, spiking and bursting. Int. J. Bifur. and Chaos 10(6), 1171-1266 (2000)

20. Jalics, J., Krupa, M., Rotstein, H.G.: Mixed-mode oscillations in a three time-scale system of odes motivated by a neuronal model. Dyn. Syst. 25(4), 445-482 (2010) 
21. Köksal Ersöz, E., Desroches, M., Krupa, M.: Synchronization of weakly coupled canard oscillators. Physica D 349, 46-61 (2017)

22. Köksal Ersöz, E., Desroches, M., Mirasso, C.R., Rodrigues, S.: Anticipation via canards in excitable systems (2018). Submitted

23. Kramer, M.A., Traub, R.D., Kopell, N.: New dynamics in cerebellar purkinje cells: torus canards. Phys. Rev. Lett. 101(6), 068103 (2008)

24. Krupa, M., Popovic, N., Kopell, N., Rotstein, H.G.: Mixed-mode oscillations in a three time-scale model for the dopaminergic neuron. Chaos 18(49), 015106 (2008)

25. Krupa, M., Szmolyan, P.: Relaxation oscillation and canard explosion. J. Differ. Equ. 174(2), 312-368 (2001)

26. Krupa, M., Wechselberger, M.: Local analysis near a folded saddle-node singularity. J. Differ. Equ. 248, 2841-2888 (2010)

27. Mitry, J., McCarthy, M., Kopell, N., Wechselberger, M.: Excitable neurons, firing threshold manifolds and canards. J. Math. Neurosci. 3(12) (2013)

28. Moehlis, J.: Canards for a reduction of Hodgkin-Huxley equations. J. Math. Biol. 52, 141-153 (2006)

29. Nowacki, J., Osinga, H.M., Tsaneva-Atanasova, K.: Dynamical systems analysis of spikeadding mechanisms in transient bursts. J. Math. Neurosci. 2(7) (2012)

30. O'Donovan, M.J.: The origin of spontaneous activity in developing networks of the vertebrate nervous system. Curr. Opin. Neurobiol. 9, 94-104 (1999)

31. O'Donovan, M.J., Chub, N.: Population behavior and self-organization in the genesis of spontaneous rhythmic activity by developing spinal networks. Semin. Cell Dev. Biol. 8, 21-28 (1997)

32. Osinga, H.M., Tsaneva-Atanasova, K.: Dynamics of plateau bursting in dependence on the location of its equilibrium. J. Neuroendocrinol. 22(12), 1301-1314 (2012)

33. Rinzel, J.: Bursting oscillations in an excitable membrane model. In: B.D. Sleeman, R.J. Jarvis (eds.) Ordinary and partial differential equations (Proceedings of the Eighth Conference held at Dundee, Scotland, June 25-29, 1984), Lecture Notes in Mathematics, vol. 1511, pp. 304-316. Springer (1985)

34. Rinzel, J.: A formal classification of bursting mechanisms in excitable systems. In: E. Teramoto, M. Yumaguti (eds.) Mathematical topics in population biology, morphogenesis and neurosciences (Proceedings of an International Symposium held in Kyoto, November 10-15, 1985), Lecture Notes in Biomathematics, vol. 71, pp. 267-281. Springer (1987)

35. Rinzel, J.: A formal classification of bursting mechanisms in excitable systems. In: International Congress of Mathematicians, Berkeley, California, USA, August 3-11, 1986, vol. II, pp. 1578-1593. American Mathematical Society (1987)

36. Rubin, J., Wechselberger, M.: Giant squid-hidden canard: the 3D geometry of the Hodgkin-Huxley model. Biol. Cybern. 97, 5-32 (2007)

37. Rubin, J., Wechselberger, M.: The selection of mixed-mode oscillations in a HodgkinHuxley model with multiple timescales. Chaos 18, 015105 (2008)

38. Szmolyan, P., Wechselberger, M.: Canards in $\mathbb{R}^{3}$. J. Differ. Equ. 177, 419-453 (2001)

39. Tabak, J., O’Donovan, M.J., Rinzel, J.: Differential control of active and silent phases in relaxation models of neuronal rhythms. J. Comput. Neurosci. 21(3), 307-328 (2006)

40. Tabak, J., Rinzel, J., O’Donovan, M.J.: The role of activity-dependent network depression in the expression and self-regulation of spontaneous activity in the developing spinal cord. J. Neurosci. 21(22), 8966-8978 (2001)

41. Tabak, J., Senn, W., O'Donovan, M.J., Rinzel, J.: Modeling of spontaneous activity in developing spinal cord using activity-dependent depression in an excitatory network. J. Neurosci. 20(8), 3041-3056 (2000)

42. Terman, D.: Chaotic spikes arising from a model of bursting in excitable membranes. SIAM J. Appl. Math. 51(5), 1418-1450 (1991)

43. Tsaneva-Atanasova, K., Osinga, H.M., Riess, T., Sherman, A.: Full system bifurcation analysis of endocrine bursting models. J. Theor. Biol. 284(4), 1133-1146 (2010)

44. Wechselberger, M.: Existence and bifurcation of canards in $\mathbb{R}^{3}$ in the case of a folded node. SIAM J. Appl. Dyn. Syst. 4(1), 101-139 (2005)

45. Wechselberger, M., Mitry, J., Rinzel, J.: Canard theory and excitability. In: P.E. Kloeden, Pötzsche (eds.) Nonautonomous Dynamical Systems in the Life Sciences, Lecture Notes in Mathematics. Springer International Publishing Switzerland 2013 (2012) 
46. Wilhelm, J.C., Rich, M.M., Wenner, P.: Compensatory changes in cellular excitability, not synaptic scaling, contribute to homeostatic recovery of embryonic network activity. Proc Natl Aced Sci U S A 106(16), 6760-6765 (2009) 